\title{
Synaptic Actions of Amyotrophic Lateral Sclerosis- Associated G85R-SOD1 in the Squid Giant Synapse
}

\author{
(1) Yuyu Song ${ }^{1,2,3,4}$
}

https://doi.org/10.1523/ENEURO.0369-19.2020

${ }^{1}$ Department of Genetics, Yale School of Medicine, Howard Hughes Medical Institute, New Haven, CT 06510,

${ }^{2}$ Laboratory of Systems Pharmacology, Program in Therapeutic Science, Harvard Medical School, Boston, MA 02115,

${ }^{3}$ Department of Neurology, Massachusetts General Hospital, Charlestown, MA 02129, and ${ }^{4}$ Marine Biological Laboratory, Woods Hole, MA 02543

\begin{abstract}
Altered synaptic function is thought to play a role in many neurodegenerative diseases, but little is known about the underlying mechanisms for synaptic dysfunction. The squid giant synapse (SGS) is a classical model for studying synaptic electrophysiology and ultrastructure, as well as molecular mechanisms of neurotransmission. Here, we conduct a multidisciplinary study of synaptic actions of misfolded human G85R-SOD1 causing familial amyotrophic lateral sclerosis (ALS). G85R-SOD1, but not WT-SOD1, inhibited synaptic transmission, altered presynaptic ultrastructure, and reduced both the size of the readily releasable pool (RRP) of synaptic vesicles and mobility from the reserved pool (RP) to the RRP. Unexpectedly, intermittent high-frequency stimulation (iHFS) blocked inhibitory effects of G85R-SOD1 on synaptic transmission, suggesting aberrant $\mathrm{Ca}^{2+}$ signaling may underlie G85R-SOD1 toxicity. Ratiometric $\mathrm{Ca}^{2+}$ imaging showed significantly increased presynaptic $\mathrm{Ca}^{2+}$ induced by G85R-SOD1 that preceded synaptic dysfunction. Chelating $\mathrm{Ca}^{2+}$ using EGTA prevented synaptic inhibition by G85R-SOD1, confirming the role of aberrant $\mathrm{Ca}^{2+}$ in mediating G85R-SOD1 toxicity. These results extended earlier findings in mammalian motor neurons and advanced our understanding by providing possible molecular mechanisms and therapeutic targets for synaptic dysfunctions in ALS as well as a unique model for further studies.
\end{abstract}

Key words: amyotrophic lateral sclerosis associated; calcium; giant synapse; neurodegeneration; SOD1; synaptic vesicles

\section{Significance Statement}

The squid giant synapse (SGS) presents one of the few mature nervous systems in situ that mimics mammalian neuromuscular junctions, while allowing precise experimental manipulations and live measurement with superior spatial and temporal resolution. Applying these unique features to studying the molecular mechanisms of amyotrophic lateral sclerosis (ALS), a devastating adult-onset neurodegenerative disease without cure, offers clues to understand the pathogenesis of the disease. Our results demonstrating synaptic dysfunction caused by ALS-associated mutant SOD1 protein and its underlying molecular pathways may suggest a novel approach to an effective therapeutic intervention as well as identify biomarkers for early diagnosis. Furthermore, the altered synaptic vesicle behavior and $\mathrm{Ca}^{2+}$ dynamics revealed through the perturbation of neurotransmission by ALS extends our understanding of fundamental synaptic physiology at both molecular and cellular levels.

Received September 14, 2019; accepted January 27, 2020; First published March 18, 2020.

The authors declare no competing financial interests.
Author contributions: Y.S. designed research; Y.S. performed research; Y.S. contributed unpublished reagents/analytic tools; Y.S. analyzed data; Y.S. wrote the paper. 


\section{Introduction}

Amyotrophic lateral sclerosis (ALS) is a fatal adult-onset neuromuscular disease with dysfunction and loss in both upper and lower motor neurons, resulting in progressive muscle weakness and atrophy, eventual paralysis, and death usually within three to five years after initial diagnosis. Inherited forms of ALS represent roughly $10 \%$ of cases, with similar clinical symptoms to sporadic ALS patients. Unfortunately, there is still no effective cure for ALS. To enable therapeutic intervention at an early stage of the disease, the mechanisms underlying ALS and presymptomatic biomarkers for early diagnosis must be identified.

Mutations in $\mathrm{Cu} / \mathrm{Zn}$ superoxide dismutase (SOD1) are responsible for $\sim 25 \%$ of familial ALS cases, but SOD1 is expressed in many cell types where it removes superoxide radicals and is a component of redox signaling pathways. ALS-associated mutations induce a conformational change within SOD1 due to protein misfolding (Bruijn et al., 1998) and aberrant interactions with other proteins (Pasinelli et al., 2004; Urushitani et al., 2006). Genetic knock-out of SOD1 does not produce motor deficits seen in ALS, indicating that SOD1 mutations confer a toxic gain-of-function (Bunton-Stasyshyn et al., 2015; Taylor et al., 2016; Ghasemi and Brown, 2018). A glycine-to-arginine substitution at residue 85 (G85R) is one highly studied ALS-associated mutation: G85R-SOD1 is incapable of dimerizing normally and instead misfolds into soluble monomers or oligomers, as well as insoluble aggregates that are toxic to motor neurons (Wang et al., 2009a,b).

To characterize G85R-SOD1 toxicity specific to neurons, various forms of misfolded G85R-SOD1-YFP were perfused into isolated axoplasm from squid giant axons (Song et al., 2013), and both soluble monomers and oligomers inhibited axonal transport from cell body to synapse through aberrant activation of signaling pathways (Song et al., 2013). Although G85R-SOD1 inhibition of anterograde transport of synaptic components could lead to synaptic dysfunction, questions remained as to whether G85R-SOD1 has a direct effect on synaptic transmission. Impairment of neuromuscular junctions is detected early in patients and animal models (Moloney et

This work was supported by the Grass Foundation, the Howard Hughes Medical Institute, the Massachusetts General Hospital Jack Satter Foundation, the Harvard University ALS and Alzheimer's Endowed Research Fund, and Harvard Brain Science Initiative.

Acknowledgements: The author thanks Dr. Arthur Horwich, Dr. George Augustine, Dr. Scott Brady, Dr. Rodolfo Llinas, and Dr. Wayne Fenton for their valuable suggestions and critical evaluation of the experiments and the manuscript. SOD1 proteins were gifts from the Horwich laboratory, prepared by Dr. Weiming Ni and Dr. Wayne Fenton. The author also received help from Dr. Jorge E. Moreira and Dr. Pri Comassio with EM data acquisition and analysis. Finally, the author would like to sincerely thank all the 2014 Grass Fellows, Dr. Alberto Pereda (director), Dr. Matthew Kittelberger (associate director), Dr. Felix Schweizer (president), the Marine Resources Center (MRC) at the Marine Biological Laboratory, and all Grass Trustees, without whose support and encouragement the project wouldn't have been possible.

Correspondence should be addressed to Yuyu Song at yuyu_song@ hms.harvard.edu or yuyu.song@aya.yale.edu.

https://doi.org/10.1523/ENEURO.0369-19.2020 Copyright @ 2020 Song

This is an open-access article distributed under the terms of the Creative Commons Attribution 4.0 International license, which permits unrestricted use, distribution and reproduction in any medium provided that the original work is properly attributed. al., 2014), consistent with a "dying-back" neuropathy where synaptic dysfunction and loss is a primary pathology eventually leading to neuronal death. Decreased synaptic vesicle numbers and reduced presynaptic proteins in presynaptic terminals of several ALS animal models further suggest a presynaptic effect (Wang et al., 2009a). These changes are seen before motor deficits are detectable, suggesting that synapses could be a primary target of G85R-SOD1 (Frey et al., 2000; Fischer et al., 2004; Hegedus et al., 2007; Sakowski et al., 2012).

Synaptic transmission is altered in ALS mouse models and induced Pluripotent Stem Cells (iPSCs)-derived motor neurons, raising key questions: how do ALS-associated proteins alter synaptic functions? Can synaptic function be restored? Unfortunately, the complexity of mouse motor systems, chronic nature of disease pathology, small size of presynaptic domains, and difficulty of targeting synapses limit utility of mouse and cell culture models in addressing questions about presynaptic mechanisms.

The squid giant synapse (SGS), which mimics neuromuscular junctions with similar ion channel compositions and neurotransmission machinery (Katz and Miledi, 1967, 1977), was used to evaluate presynaptic and postsynaptic actions of G85R-SOD1 here. The large size of squid giant presynaptic terminals permits experimental manipulations such as protein microinjection, direct electrophysiological measurements of transmission, current and voltage clamping, and $\mathrm{Ca}^{2+}$ imaging, facilitating detailed studies of presynaptic molecular mechanisms (Bloedel et al., 1966; Llinas et al., 1980, 1994; Augustine and Charlton, 1986; Llinás et al., 1985; Augustine et al., 1988, 2006; Augustine, 1990; Lin et al., 1990; Fukuda et al., 1995; Sugimori et al., 1998). The squid synapse is particularly suitable for studying ALS, because it is (1) fast-transmitting with high release capacity, similar to synapses affected in ALS; and (2) enriched in ion channels (e.g., $\mathrm{Ca}^{2+}$ ), neurotransmitters (e.g., glutamate) and receptors (e.g., AMPAR) implicated in ALS pathology.

Here, we microinjected G85R-SOD1 in presynaptic terminals, recorded both presynaptic and postsynaptic membrane potentials with and without high-frequency stimulations (HFSs), performed live ratiometric $\mathrm{Ca}^{2+}$ imaging, and examined synaptic vesicle morphology/distribution by electron microscopy (EM). Correlated functional and structural studies demonstrated the acute synaptic inhibition by G85R-SOD1 as evidenced by diminished postsynaptic potentials (PSPs), decreased synaptic vesicle number at active zones (AZs), and reduced synaptic mobilization from reserved (RP) to readily releasable pool (RRP) within $30 \mathrm{~min}$ after G85R-SOD1 injection. Unexpectedly, while continuous trains of HFS depleted synaptic vesicle availability and enhanced synaptic inhibition by G85RSOD1, intermittent HFS (iHFS) applied every 30 min before and during G85R-SOD1 infusion kept synapses firing normally for $7 \mathrm{~h}$. Given the effects of HFS on presynaptic $\mathrm{Ca}^{2+}$, we tested for aberrant $\mathrm{Ca}^{2+}$ signaling by ratiometric $\mathrm{Ca}^{2+}$ imaging with presynaptic fura-2. $\mathrm{Ca}^{2+}$ increased throughout terminals including in "palm" regions before presynaptic axons branch, which lack plasmalemmal $\mathrm{Ca}^{2+}$ channels. Reducing intracellular $\mathrm{Ca}^{2+}$ by EGTA rescued synaptic function and restored normal structure in the presence of G85R-SOD1 as did iHFS. These results are 
consistent with and extend findings in mammalian nervous systems where neurons with lower $\mathrm{Ca}^{2+}$ buffering capacity are selectively affected in ALS and these neurons showed $\mathrm{Ca}^{2+}$ imbalances presymptomatically (Grosskreutz et al., 2010). Understanding sources of increased $\mathrm{Ca}^{2+}$ and molecular mechanisms underlying $\mathrm{Ca}^{2+}$ activation as well as how iHFS rescues synaptic function will provide unique insights into synaptic dynamics and a possible molecular basis for synaptic pathology in human ALS patients.

\section{Materials and Methods}

\section{Proteins and reagents}

G85R-SOD1-His, WT-SOD1-His, G85R-SOD1-HisYFP, and WT-SOD1-His-YFP were produced in E. coli BL21/DE3 cells under pET vectors, and purified on Talon resin first and then by chromatography on MonoQ 10/10, in the Horwich Laboratory as described previously (Song et al., 2013). All chemicals used were American Chemical Society quality or better, from Sigma and Invitrogen (now Fisher Scientific). For microinjection, proteins and reagents were dissolved in $200 \mathrm{~mm} \mathrm{KCl,} 100 \mathrm{~mm}$ taurine, $250 \mathrm{~mm}$ K-isethionate, and $50 \mathrm{~mm}$ K-HEPES, $\mathrm{pH}$ 7.4). Tetramethylrhodamine-dextran ( $3 \mathrm{kDa}$, Invitrogen) was used for co-injection with non-fluorescent reagents to visualize and monitor the injection.

\section{SGS preparation and electrophysiology setup}

The giant squid synapse is formed between the terminal finger of second order axons of the pre-nerve and the third order axon of the last stellate nerve, which is the giant axon used for axonal transport studies as well as for classical voltage-clamp studies of ion channels (Young and Keynes, 2005). Following a standard protocol (Llinas et al., 1980, 1981, 1994; Llinás et al., 1985; Augustine and Charlton, 1986; Augustine et al., 1988, 2006; Augustine, 1990; Smith et al., 1993), stellate ganglia of small female Loligo pealeii (RRID: SCR_002864, Marine Biological Laboratory) were removed from the mantle carefully and rapidly under running seawater, tied off at each end of the presynaptic and postsynaptic axons, isolated from the sheath and connective tissues, pinned with fine cactus needles to a thin-layer of Sylgard on the bottom of a 35$\mathrm{mm}$ Petri dish chamber, superfused continuously with oxygenated squid saline ( $455 \mathrm{~mm} \mathrm{NaCl}, 54 \mathrm{~mm} \mathrm{MgCl}, 11$ $\mathrm{mm} \mathrm{CaCl}, 10 \mathrm{~mm} \mathrm{KCl}, 3 \mathrm{~mm} \mathrm{NaHCO}_{3}$, and $10 \mathrm{~mm} \mathrm{HEPES}$, $\mathrm{pH} 7.2$ ) at $10-15^{\circ} \mathrm{C}$.

The most distal digit of the presynaptic axons and the most medial fiber, also known as the giant axon, form the giant synapse (Fig. 1A). One microelectrode for injecting was inserted in the presynaptic axon to inject current at $0.033 \mathrm{~Hz}$ for basal stimulation and at $50 \mathrm{~Hz}$ for HFS (each pulse is $2 \mu \mathrm{A}$ for $2 \mathrm{~ms}$ for basal stimulation and $2 \mu \mathrm{A}$ for $1 \mathrm{~ms}$ for HFS), near the palm where the second order axon enters the ganglion to branch and form multiple synapses with postsynaptic axons. At the terminal of the most medial branch, a second microelectrode was inserted to presynaptically inject proteins and reagents of interest as well as recording the presynaptic membrane potentials. Finally, at the postsynaptic terminal of the giant synapse, a third microelectrode was inserted near the medial presynaptic digit to record the postsynaptic membrane potentials. Electrodes 1 and 3 were filled with $3 \mathrm{M} \mathrm{KCl}$ while the second microelectrode allowed the injection of $50 \mu \mathrm{M}$ SOD1 proteins or reagents of interest in $100 \mathrm{~mm} \mathrm{KCl}$ (Smith et al., 1993) at $0.1 \mathrm{~Hz}$ (each injection was $50 \mathrm{psi}$ for $250 \mathrm{~ms}$ ). The injection efficiency of SOD1 proteins was monitored by a fluorescence microscope. All synapses were injected with the same pressure for the same amount of time during data acquisition, and the final mean fluorescence intensities in the presynaptic terminals were measured and compared between samples to ensure comparable levels of infused proteins.

\section{Electrophysiology data acquisition and analysis}

Both presynaptic potential and PSP were recorded using sharp microelectrodes with an Axoclamp-2A amplifier (Axon Instrument) and data analyzed using Labview (National Instruments) software (Yulong Li). Raw waveforms were used to calculate PSP slope using the same parameters for all experiments. For data acquired under HFS, PSP slopes were analyzed, integrated, plotted, and fitted linearly using the last 50 time points to derive the vesicle mobilization rate (slope of the linear fit) and the RRP size (intersection with the $y$-axis).

\section{Ratiometric $\mathrm{Ca}^{2+}$ imaging}

We dissolved $1 \mathrm{~mm}$ fura-2 (pentapotassium salt, ThermoFisher F1200, CHEBI:52 081), a $\mathrm{Ca}^{2+}$ indicator, in $100 \mathrm{~mm} \mathrm{KCl}$ and infused it into the presynaptic terminal by electrophoresis (100 nA current) through the first presynaptic electrode inserted in the palm. Fura-2 infusion continued for $10 \mathrm{~min}$, and the synapse was allowed to equilibrate for $30 \mathrm{~min}$ before presynaptic injection of SOD1 proteins through the second microelectrode to ensure fura-2 was diffused evenly throughout the whole presynaptic terminal at roughly $100 \mu \mathrm{m}$. Ratiometric live $\mathrm{Ca}^{2+}$ imaging was performed every $30 \mathrm{~s}$ with ratiometric images taken at Ex360 $\mathrm{nm}$ and Ex390 nm assisted by an ultra-high-speed wavelength switching $\lambda$ DG-4/5 xenon arc lamp system. Ratios at various locations (A) were calculated as (fluorescent intensity $\left.{ }^{360 \mathrm{~nm}}\right) /\left(\right.$ fluorescent intensity ${ }^{390 \mathrm{~nm}}$ ) as a measure of intracellular $\mathrm{Ca}^{2+}$ concentration (Smith et al., 1993). In vitro calibration was performed by mixing squid axoplasm with equal volumes of buffer containing $5 \mu \mathrm{m}$ fura-2, $400 \mathrm{~mm}$ $\mathrm{KCl}$, and $40 \mathrm{~mm} \mathrm{Na-HEPES} \mathrm{(pH} \mathrm{7.2).} \mathrm{Three} \mathrm{doses} \mathrm{of} \mathrm{CaCl}_{2}$ were set by adding $20 \mathrm{~mm}$ EGTA (low $\mathrm{Ca}^{2+},<10^{-8} \mathrm{M}$ ), or $20 \mathrm{~mm}$ EGTA + $13.3 \mathrm{~mm} \mathrm{CaCl}_{2}$ (intermediate $\mathrm{Ca}^{2+}$, $\sim 6.7 \times 10^{-7} \mathrm{M}$ ), or $20 \mathrm{~mm} \mathrm{CaCl}_{2}\left(\right.$ high $\mathrm{Ca}^{2+}$ ). Various ratios under each condition were measured using the same optics and the background noise was subtracted. The dose response curve was obtained to allow the determination of $\mathrm{Kd}^{\prime}$ and the two ratios. Rmax was defined as the ratio value at saturating $\mathrm{Ca}^{2+}$ level, and $\mathrm{Rmin}$ was defined as the ratio value at the minimal $\mathrm{Ca}^{2+}$ concentration.

\section{EM}

After recordings, synapses were fixed in 4\% glutaraldehyde and $2 \%$ paraformaldehyde in $0.1 \mathrm{M}$ cacodylate buffer for $12 \mathrm{~h}$ at $4^{\circ} \mathrm{C}$, then washed in $0.1 \mathrm{M}$ cacodylate 
A

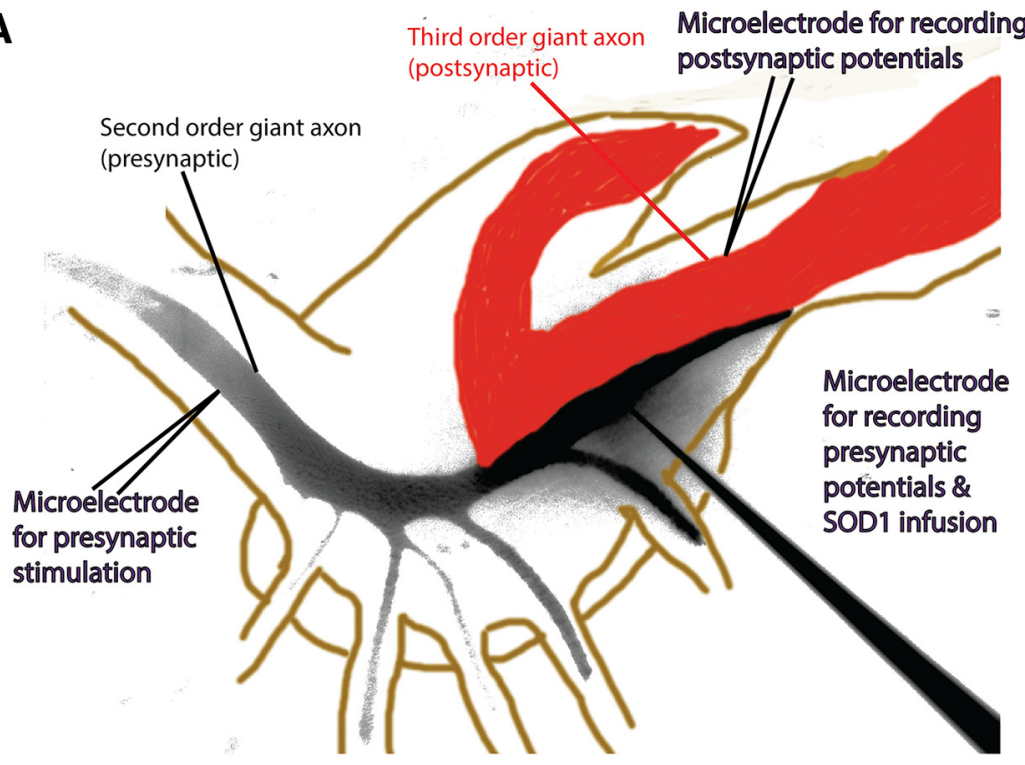

B

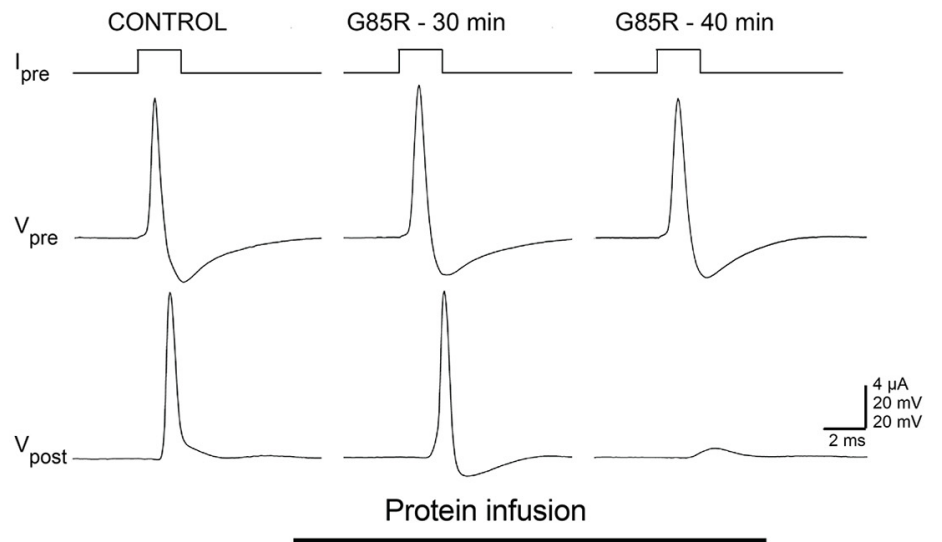

C

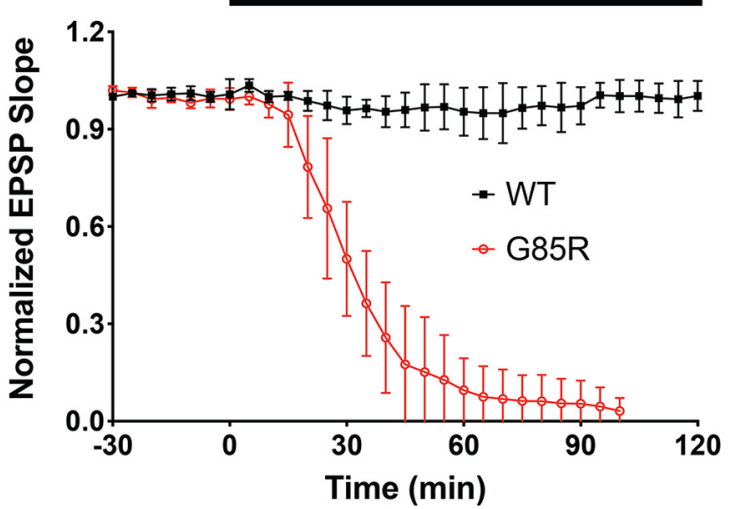

Figure 1. Presynaptic infusion of G85R-SOD1-YFP inhibited synaptic transmission. $\boldsymbol{A}$, Diagram of experimental setup. Presynaptic second order giant axon branch (black) and postsynaptic third order giant axon (red) form the giant synapse in the stellate ganglion. Two electrodes were inserted in the presynaptic axon, one at the palm to deliver electronic stimulation at $0.03 \mathrm{~Hz}$ (each pulse is $2 \mu \mathrm{A}$ for $2 \mathrm{~ms}$ ) and the other in the presynaptic terminal for recording presynaptic potentials as well as injecting $50 \mu \mathrm{m}$ SOD1 proteins and reagents of interest at $0.1 \mathrm{~Hz}$, each injection was $50 \mathrm{psi}$ for $250 \mathrm{~ms}$. The third electrode was inserted in the postsynaptic axon to record PSPs. B, Under current clamping condition, G85R-SOD1-YFP-injected synapses showed reductions in PSP ( $\left.\mathrm{V}_{\text {post }}\right)$ within $30 \mathrm{~min}$ and a failure to elicit a postsynaptic action potential in $40 \mathrm{~min}$, compared with the control before injection. $\boldsymbol{C}$, EPSP slopes of WT-SOD1-YFP-injected synapses $(n=5)$ and G85R-SOD1-YFP-injected synapses $(n=8)$ were normalized to the initial time point (-30 $\mathrm{min}$ ), $30 \mathrm{~min}$ before the beginning of SOD1 injections. Averaged EPSP slopes were plotted with error bars showing standard error (SE). The thick black bar indicates the duration of SOD1 infusion starting at time 0. G85R-SOD1-YFP consistently inhibited synaptic transmission as shown by reductions in the EPSP slope, while WT-SOD1-YFP infusions showed no effect on EPSPs. Control synapses infused with fluorescent dextran showed normal firing similar to the WT-SOD1-YFP-infused synapses (Extended 
continued

Data Fig. 1-1). Moreover, synaptic function gradually recovered in G85R-SOD1-YFP-infused synapses after protein infusion was stopped, excluding the possibility of physical damage of synaptic machinery due to injection (Extended Data Fig. 1-2).

buffer three times and postfixed in $1 \%$ osmium tetroxide for $2 \mathrm{~h}$ at $4^{\circ} \mathrm{C}$, followed by blocking impregnation with $2 \%$ uranyl acetate in $0.1 \mathrm{M}$ sodium acetate, $\mathrm{pH} 5.0$ for $24 \mathrm{~h}$. After washing, dehydration was performed in ethanol with increasing concentration: $50 \%$ for $10 \mathrm{~min}, 75 \%$ for $10 \mathrm{~min}, 80 \%$ for $10 \mathrm{~min}, 85 \%$ for $10 \mathrm{~min}, 95 \%$ for $10 \mathrm{~min}$, and $100 \%$ for 5 min twice, followed by propylene oxide for 5 min three times. Resin and propylene $(1: 1$ for $24 \mathrm{~h}$, $2: 1$ overnight, and $3: 1$ for $2-4 \mathrm{~h}$, and finally $100 \%$ resin overnight) infiltration was conducted before embedding in silicone molds in the oven at $62^{\circ} \mathrm{C}$ for $72 \mathrm{~h}$. Sectioning and imaging were performed following standard EM protocols (Augustine et al., 2006; Moreno et al., 2009). Vesicle density at the active zones (AZs) was determined as the number of vesicles per square micrometer. Distance between vesicles and AZs was measured and plotted as described previously (Morgan et al., 1999). All EM samples were numbered, processed, and analyzed under blinded conditions until the last step when data had to be pooled.

\section{Statistical information}

All experiments were repeated at least five times. The data were analyzed by one-way ANOVA followed by the Tukey post hoc test (or nonparametric multiple $t$ tests, without assuming consistent SD) and plotted in Prism 7 (GraphPad software). Quantitative data were plotted as mean \pm SEM, $p$ values were calculated and four statistical thresholds were marked: $p<0.00,001, p<0.0001, p<0.001$, and $p<0.005$ $(p<0.05$ indicated statistical significance). For EM analysis, nested one-way ANOVA was performed to compare WT and G85R-infused synapses (102 synapses across three synapses for each group).

\section{Results}

\section{G85R-SOD1-YFP but not WT-SOD1-YFP inhibits synaptic transmission}

To assess whether the mutant SOD1 is directly toxic to the synapse, as opposed to indirectly affecting it, e.g., by blocking axonal anterograde vesicular trafficking (Song et al., 2013), G85R-SOD1-YFP $(n=8)$ or WT-SOD1-YFP control $(n=5)$ was microinjected in the presynaptic terminal site (Fig. 1A) through the micropipette extending in from lower right; note that black color indicates the extent of YFP fluorescence after 40 min of infusion. Another microelectrode was used to pass current pulses for basal stimulation $(2 \mu \mathrm{A} \times 2 \mathrm{~ms}$, every $33 \mathrm{~s}$; Fig. $1 A$, micropipette extending in from lower left) to evoke single action potentials, detected by intracellular voltage recording simultaneously from both presynaptic and postsynaptic sites (Fig. $1 A$ for sites of recording and Fig. $1 B$, middle and bottom traces). This protocol is referred to hereafter as "basal stimulation." Measurement of voltage changes at both presynaptic and postsynaptic sites allows for assessment of the efficiency of synaptic transmission.
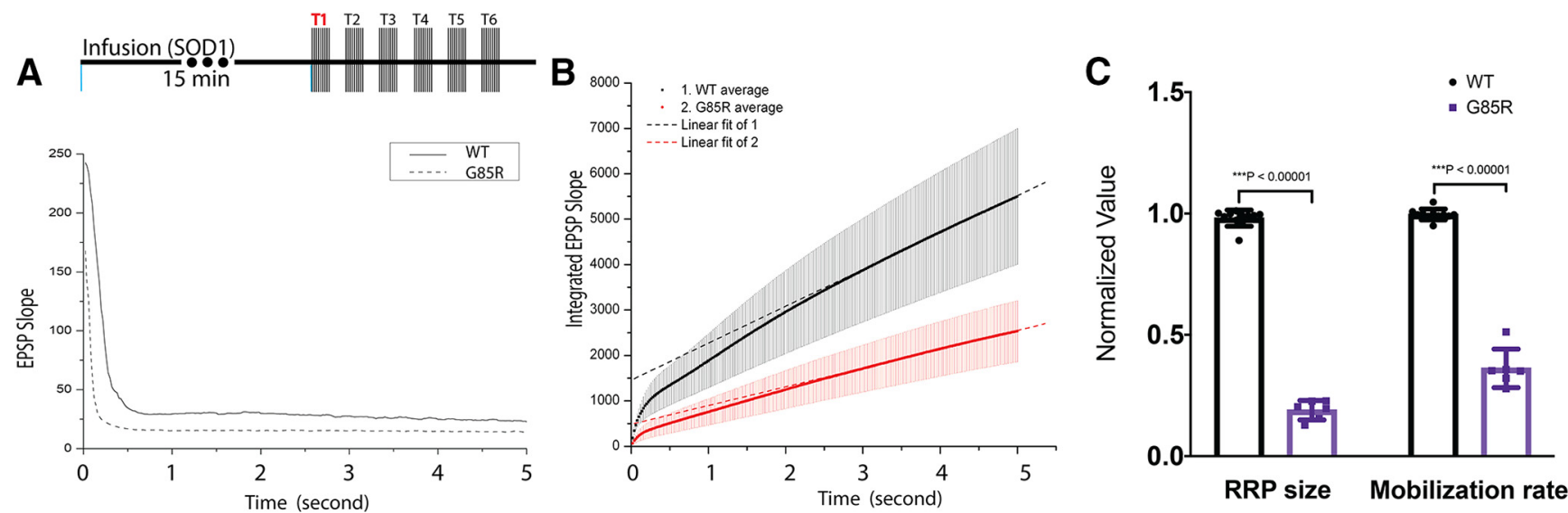

Figure 2 Presynaptic infusion of G85R-SOD1-YFP altered synaptic vesicle dynamics. $\boldsymbol{A}$, Series of six continuous trains of HFS (each train is $50 \mathrm{~Hz}$ for $5 \mathrm{~s}$ with $5 \mathrm{~s}$ between trains) was applied to the synapses infused with SOD1 proteins for 15 min before HFS trains. WT-SOD1-GFP-injected synapses showed constant EPSP slope at the beginning of each train suggesting robust neurotransmission (>200, also see Extended Data Fig. 2-1 for the sixth train). However, G85R-SOD1-YFP-injected synapse started to show reductions in synaptic transmission, as evidenced by the dramatic decrease in EPSP slopes taken during the first train both at the beginning and at the steady state, suggesting severe depletion of synaptic vesicles from both the RRPs and the RPs. This inhibition was more obvious in the sixth train (Extended Data Fig. 2-1). B, EPSPs of the first train from WT-SOD1-YFP-injected synapses (black, $n=11$ ) and from G85R-SOD1-YFP-injected synapses (red, $n=6$ ) were integrated and averaged, followed by linear fit. The intersection with $y$-axis indicated the size of the RRP and the slope indicated the mobilization rate of vesicles from the RP to RRP. C, Normalized to baseline values before the injection of SOD1-YFP, RRP size and mobilization rate from the first train were plotted as individual biological replicates ( $n=11$ for WT-SOD1-infused synapses and $n=6$ for G85R-SOD1-infused synapses) to show significant reductions in both RRP and mobilization of vesicles from the RP by G85R-SOD1-YFP, but not by WT-SOD1-YFP. 
A

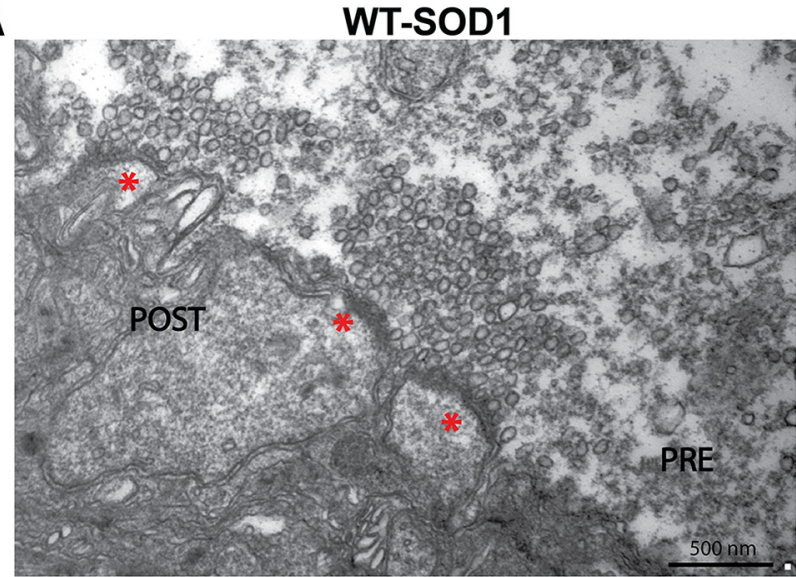

C

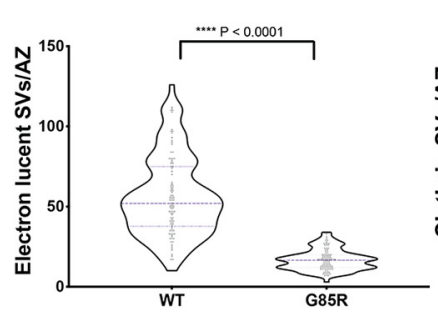

G

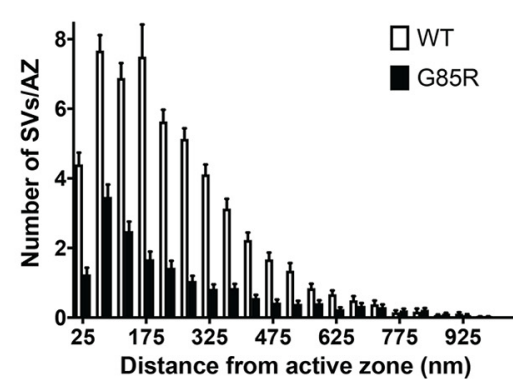

H
B

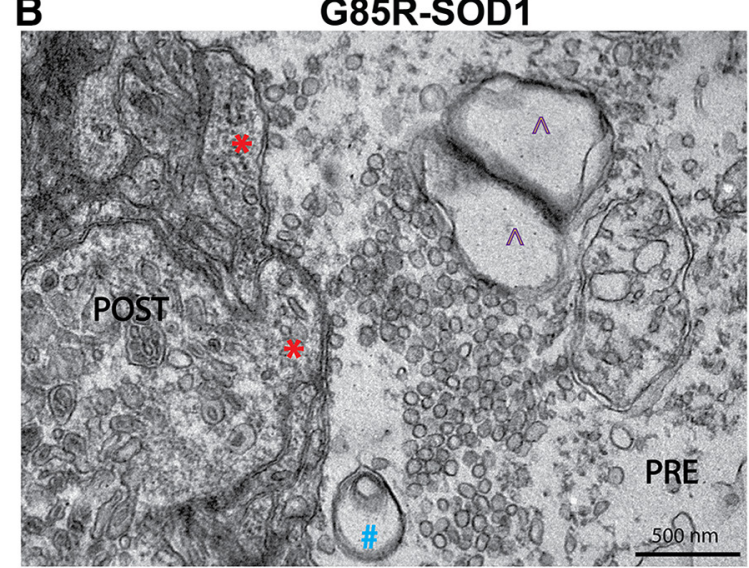

D

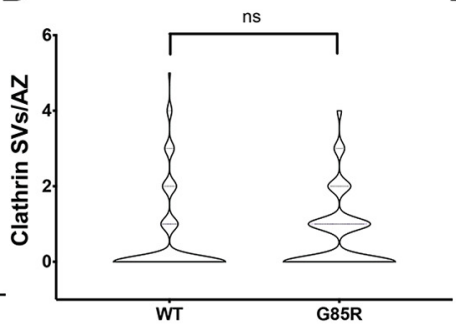

$\mathbf{F}$

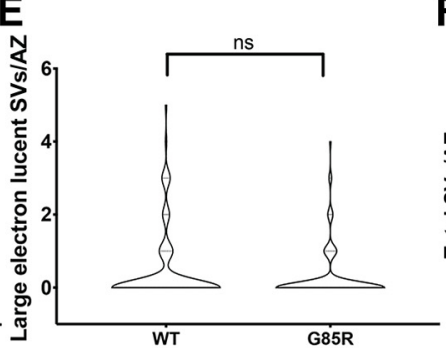

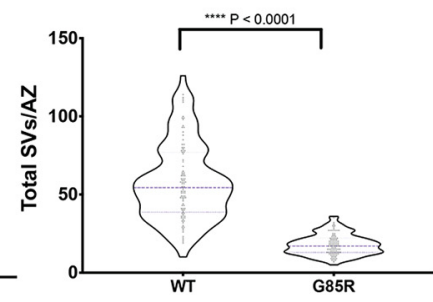

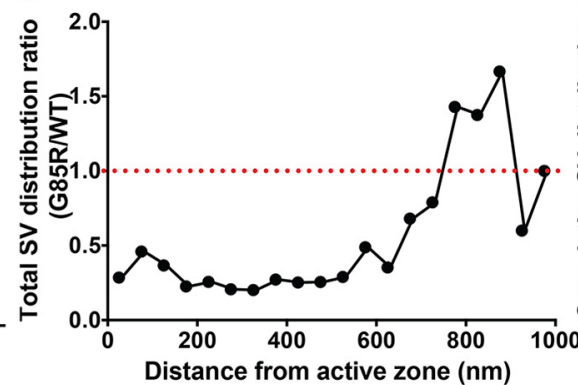

I

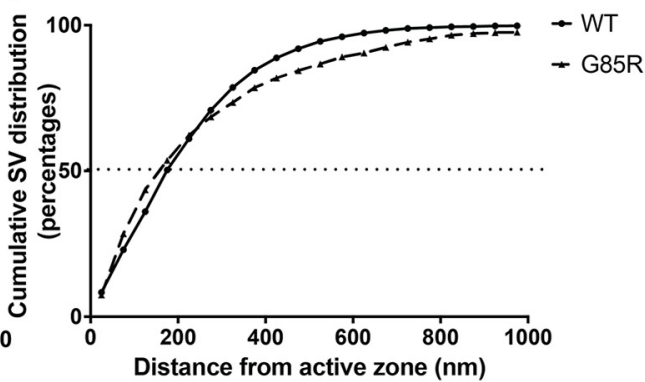

Figure 3. Presynaptic infusion of G85R-SOD1 inhibited synaptic vesicle (SV) availability. Representative EM images illustrate morphology of AZs (labeled with red $*$ ) and numbers of SVs in fixed synapses infused with WT-SOD1 $(\boldsymbol{A}, n=3)$ and G85R-SOD1 (B, $n=3$ ). G85R-SOD1-infused synapses showed vacant AZs and occasional abnormal membranous structures (indicated by blue \# and purple $\wedge^{\text {). }} . \mathbf{C}-\boldsymbol{F}$, Quantification of averaged vesicle number across all AZs from 102 WT- and 102 G85R-SOD1-YFP-infused synapses showed statistically significant reductions in the total vesicle number and in the electron lucid vesicle number by G85R-SOD1-YFP as compared with WT-SOD1. The clathrin-coated vesicles and the large electron lucid vesicles were comparable between WT- and G85R-SOD1-YFP-infused synapses. Nested one-way ANOVA was performed to compare WT and G85R-infused synapses $(p<0.0001)$ as well as across biological triplicates within each group $(p>0.05)$ ns: not significant. $G$, Averaged number of SVs per AZ were plotted along the distance from AZ (binned by $50 \mathrm{~nm}$ ). $\boldsymbol{H}$, Distance distribution plot of SVs in each $50-\mu \mathrm{m}$ bin showed a global reduction of SVs from G85R-SOD1-YFP-infused synapses regardless of the distance from the AZs. Because of the drastically reduced numbers of SVs far (>675 nm) from the AZs in both WT and G85R synapses, the reduction seemed to disappear or even be reversed, however, the differences far from the AZ may not be significant due to the substantially decreased numbers of vesicles in that area for both WT and G85R synapses. I, Cumulative SV distribution plots showed similar distribution patterns of vesicles in WT (solid line) and G85R (dashed line) synapses, confirming the even inhibition of SV availability by G85R-SOD1 independent of the distance from the AZs.

Strikingly, after 40 min of G85R-SOD1-YFP infusion, the postsynaptic action potential was no longer detected, with only a subthreshold PSP observable (Fig. 1B, Vpost). By comparison, there was no change in the PSP with a WTSOD1-YFP infusion (Fig. 1B, control) or with fluorescent dextran alone (Extended Data Fig. 1-1). A time course study of WT-SOD1-YFP and G85R-SOD1-YFP was conducted to observe the kinetic behavior of EPSPs from basal stimulation during the respective infusions (Fig. $1 C$; note that points are shown only for every 10th pulse, i.e., at 5-min intervals). With infusion of the mutant protein, the EPSP slope began to reduce by $\sim 10 \mathrm{~min}$, and was completely lost by $\sim 60$ $70 \mathrm{~min}$. By contrast, no effect on EPSP was observed with WT-SOD1-YFP infusion for $>100 \mathrm{~min}$ (Fig. 1C). Thus, in contrast with the absence of an observable effect of WTSOD1 infusion on postsynaptic EPSP, injection of monomeric G85R-SOD1-YFP produced a substantial time-dependent inhibition of synaptic transmission. 
To rule out the possibility that synaptic defects were caused by physical damage induced by electrode impalement and microinjection, injection was stopped when postsynaptic action potentials were abolished. This allowed the diffusion of G85R-SOD1-YFP away from the synaptic terminal through the presynaptic axon. The EPSP slope started to increase $90 \mathrm{~min}$ later as the presynaptic fluorescent intensity of G85R-SOD1-YFP significantly decreased and EPSPs were fully restored in $\sim 2.5 \mathrm{~h}$ after most mutant SOD1 had diffused out of the terminal (Extended Data Fig. 1-2). Normal synaptic transmission continued for $>1 \mathrm{~h}$ until a second injection of G85RSOD1-YFP significantly inhibited synaptic transmission in the same fashion as the first injection. These results suggested that G85R-SOD1-YFP inhibits synaptic transmission at least in part by directly affecting neurotransmitter release from the synaptic terminal and this effect was reversible on the removal of G85R-SOD1-YFP from the synaptic terminal.

\section{G85R-SOD1-YFP impairs synaptic vesicle availability at the $A Z$}

To examine whether G85R-SOD1-YFP inhibited synaptic transmission by affecting synaptic vesicle pool dynamics, we microinjected G85R-SOD1-YFP or WT-SOD1-YFP for $15 \mathrm{~min}$ and then applied trains of HFS presynaptically $(50 \mathrm{~Hz}$ for $5 \mathrm{~s}$, with $5 \mathrm{~s}$ intervals between trains) and measured postsynaptic depression of EPSPs, which are proportional to the underlying EPSCs and are uncontaminated by action potentials, to determine the size of the RRP and the rate of vesicle mobilization from the reserved pool (RP). EPSP slopes during both the first train of a series trains of HFS (Fig. 2A) and the sixth train (Extended Data Fig. 2-1) were compared in WT and G85R synapses. For controls, there was a two-phase diminution of EPSP slope across the 250 spikes, whereas with G85R synapses the first phase was barely detectable during the sixth train of HFS and was significantly inhibited during the first train. The first phase is generally associated with utilization of the RRP, which appears to be smaller with the mutant, whereas the second phase is generally associated with utilization of the $\mathrm{RP}$, which appears to be present in both with significant reduction by G85R-SOD1-YFP. To further measure these pools, the EPSP slope values were continuously integrated across one train of the same synapse before infusion and one train after 30 min of WT- or G85R-SOD1-YFP infusion. The EPSPs of each condition were integrated and averaged to generate traces (Fig. 2B). Notably, the WT-SOD1 trace resembled that of the preinfusion trace (not shown), indicating that WT-SOD1 has little effect on synaptic vesicle availability and mobilization, whereas G85R-SOD1 trace exhibited integrated values that were substantially lower than those of WT-SOD1. The relative size of the RRP was determined for each condition by intersection of slope with the $y$-axis, and the mobilization rate from the RP was directly determined from the slopes (dotted lines; linear fit of the integrated EPSP slopes from the last 50 time points). The values and statistics for independent experiments are shown ( $n=11$ for WT and $n=6$ for G85R; Fig. 2C). G85RSOD1-YFP drastically reduced both the RRP size and the vesicle mobilization rate from RP to RRP as compared with WT-SOD1-YFP. Therefore, reduced RRP size and slower RP mobilization rate, could lead to the loss of synaptic transmission.

\section{EM of WT-SOD1-YFP and G85R-SOD1-YFP-infused synapses}

To visualize the synaptic vesicles directly, we conducted EM analyses of synapses infused for 40 min under basal stimulation with the respective proteins $(n=3$ for each group). The WT-SOD1-YFP-infused synapses exhibited normal morphology with abundant packing of synaptic vesicles against the presynaptic membrane, comparable to published EM data (Augustine et al., 2006) of normal SGS (Fig. $3 A$, red $*$ indicates an $\mathrm{AZ}$ ), whereas the G85R-SOD1-YFP-infused synapses reproducibly exhibited synaptic vesicles that did not abut the membrane at AZs, lying in a zone distant from it (Fig. 3B). The vesicles present in this zone also appeared generally larger, less compact in morphology and reduced in number relative to WT-SOD1 synapses. We also consistently observed additional structures, e.g., in this image, what appears to be a pre-autophagosome (labeled by blue \#) and two adjoining prelysosomes (labeled by purple ${ }^{\text {a) }}$. By contrast we did not observe such structures in WTSOD1-infused presynaptic AZs. Notably, many of the preautophagosomes appeared to contain vesicles. This potentially reflects a local mechanism to remove G85RSOD1-YFP-affected synaptic vesicles in the RRP and a subpopulation of vesicles in the RP, whereas, interestingly, the most remote RP pools appear to be spared, suggesting a potential source for synaptic vesicle supply, which may help restoring synaptic function if released on proper stimulations. More detailed characterization of affected vesicles was conducted by counting the number of electron lucent vesicles, clathrin-coated vesicles, and large electron lucent vesicles per AZ as described previously (Morgan et al., 1999; Fig. 3C-F). Nested ANOVAs suggested that while clathrin-coated vesicles did not seem to be affected by G85R-SOD1, the total number of available synaptic vesicles was dramatically reduced. A cumulative measurement of AZs (102 across three biological repeats per group) infused with WT-SOD1 or G85RSOD1 (binned by $50 \mu \mathrm{m}$ in distance) showed that such reductions seem to be evenly distributed in the RRP regardless of the distance from the membrane (Fig. 3G-l). Because of the limited number of vesicles further away from the AZs $(>675 \mu \mathrm{m})$ in both groups, the increase in the relative vesicle distribution in that vicinity may not be statistically different between the G85R-SOD1-infused synapses and the WT-SOD1-infused synapses. This is further confirmed by the cumulative vesicle distribution curves (without binning) in WT and G85R-SOD1 synapses (Fig. 3/).

\section{Prevention of G85R-SOD1-YFP-associated synaptic deficits by iHFS}

Unexpectedly, when a single train of $\mathrm{HFS}(50 \mathrm{~Hz}$ for $5 \mathrm{~s})$ was applied presynaptically every $30 \mathrm{~min}$, G85R-SOD1- 
A

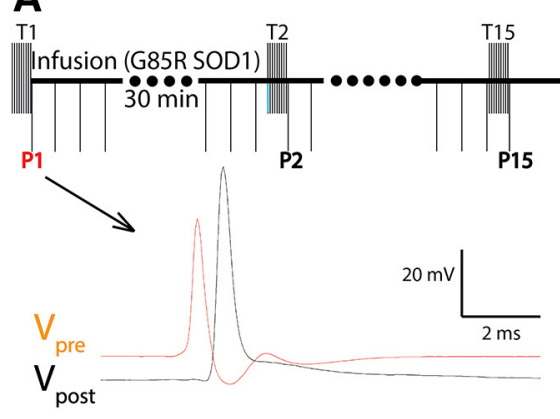

D

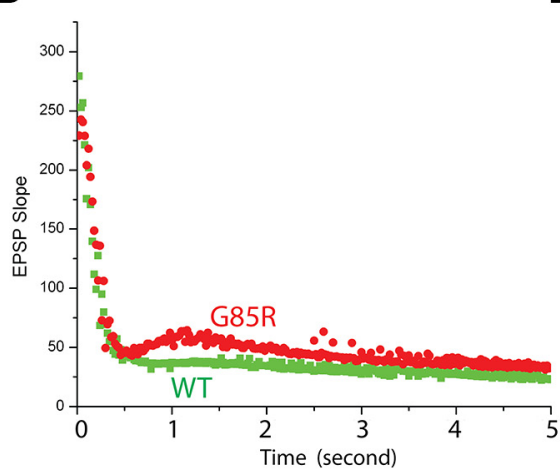

B

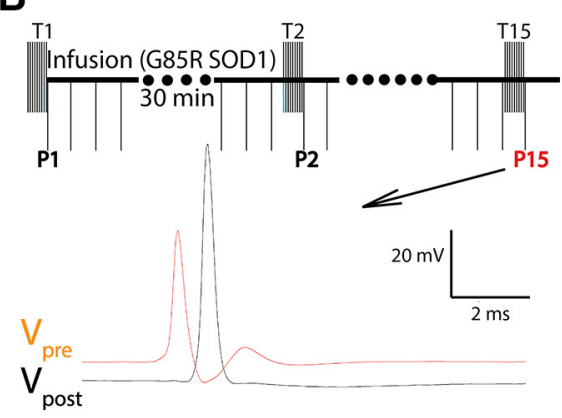

E

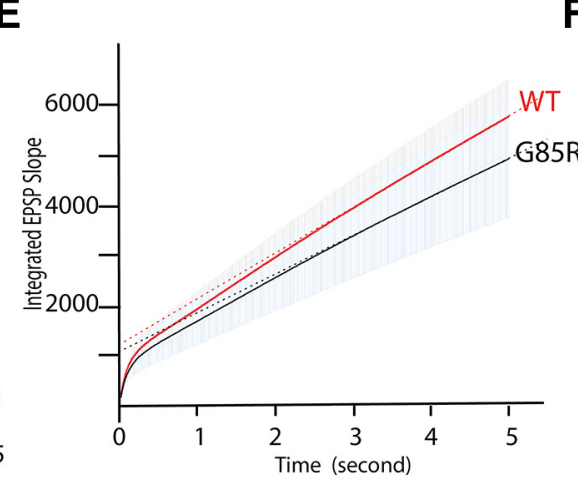

C

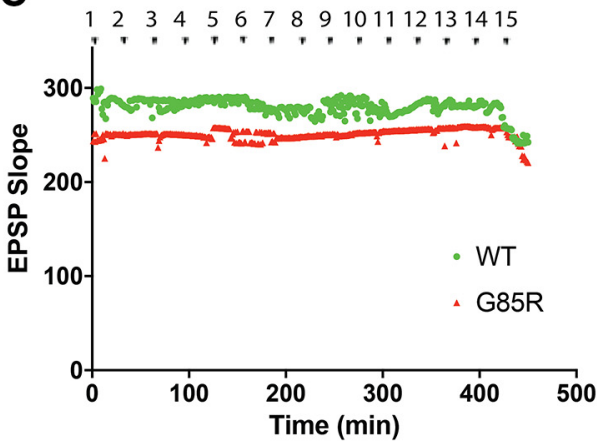

Figure 4. iHFS prevented synaptic transmission deficits caused by G85R-SOD1-YFP. A, iHFS (5 s of $50 \mathrm{~Hz}$ applied every $30 \mathrm{~min}$ ) was first applied to the synapse at time 0 before G85R injection, both presynaptic and postsynaptic membrane potentials were recorded 1 min after HFS (P1). B, If iHFS trains were applied every 30 min before and during continuous SOD1-YFP infusion into the synapse, G85R-SOD1 no longer inhibited synaptic transmission and the synapse continued to fire even after $7 \mathrm{~h}$ without significant changes in either presynaptic or postsynaptic membrane potentials (P15). C, EPSP slopes remained constant and comparable under basal stimulation with iHFS in WT and G85R synapses. $\boldsymbol{D}$, EPSP slope from one single train of iHFS showed no significant difference between WT and G85R synapses except for occasional augmentation seen in G85R-SOD1-YFP-infused synapses. E, To evaluate the vesicle dynamics, EPSP slopes were measured and integrated during HFS trains applied either with 2 h WT- or G85RSOD1-YFP infusion in the presence of iHFS every $30 \mathrm{~min}(n=6)$. There were no significant differences in RRP size and mobilization rate of vesicles trafficking from RP to RRP by G85R-SOD1-YFP. $\boldsymbol{F}$, EM showed normal presynaptic structure with normal numbers of vesicles at the AZ (indicated by red asterisk) in G85R-SOD1-YFP-infused synapses when iHFS was applied. Interestingly, in three out of five synapses, HFS restored firing in G85R-SOD1-YFP-infused synapses, where EPSPs were significantly inhibited (Extended Data Fig. 4-1).

YFP-infused synapses, otherwise receiving continuous basal stimulation, maintained constant EPSPs for over $8 \mathrm{~h}$ (Fig. $4 A-C$ ). This contrasts with the finding (Fig. 1) that in the setting of G85R-SOD1-YFP infusion, continuous basal stimulation leads to steady decline and then complete loss of EPSPs within 40-60 min. This also contrasts with the finding (Fig. 2) that continuous HFS trains with 5$s$ intervals also inhibited synaptic transmission by depletion of the RRP. Correlating with maintenance of healthy physiology, synaptic vesicle dynamics appeared to be normal, as demonstrated by presence of the first phase of EPSP slope during a single $50-\mathrm{Hz}$ train, reflecting presence of an RRP resembling wild type (Fig. 4D). Additionally, the integrated EPSP slope averaged across multiple $50-\mathrm{Hz}$ trains now resembled that of WT-SOD1-YFP synapses $(n=6$ for each; Fig. 4E). Finally, in EM analyses (Fig. 4F), consistent with presence of a normal RRP, vesicles were observed abutting the AZ after iHFS was applied to a synapse infused with G85R-SOD1-YFP for $6 \mathrm{~h}$. These results indicate that iHFS is able to preserve the RRP in the face of G85R-SOD1-YFP infusion. Interestingly, in G85R-SOD1injected synapses (three out of five) with dramatic decreases in the slope of the PSP but remaining $>50$, application of one HFS train restored EPSP slope and the RRP size, without rescuing vesicle mobilization rate (Extended Data Fig. 4-1). However, in the other two synapses where PSP slopes dropped to below 50 , adding one HFS further reduced them to almost 0 . This suggests that to achieve the rescuing effects, the timing of the HFS application with regard to existing synaptic strength is important. However, the underlying molecular mechanism of this rescuing effect is unclear and requires further study. Altogether, the intermittent stimulation reverses toxicity of the misfolded protein by restoring synaptic vesicle availability at the AZ.

\section{G85R-SOD1 induces $\mathrm{Ca}^{2+}$ increase in the presynaptic terminal}

Intrigued by the rescuing effect of iHFS in G85R-SOD1infused synapses, we examined potential mechanisms. Since iHFS is known to alter $\mathrm{Ca}^{2+}$ dynamics, we wondered if aberrant $\mathrm{Ca}^{2+}$ signaling may underlie G85RSOD1-YFP toxicity. To test this possibility, we performed 
A

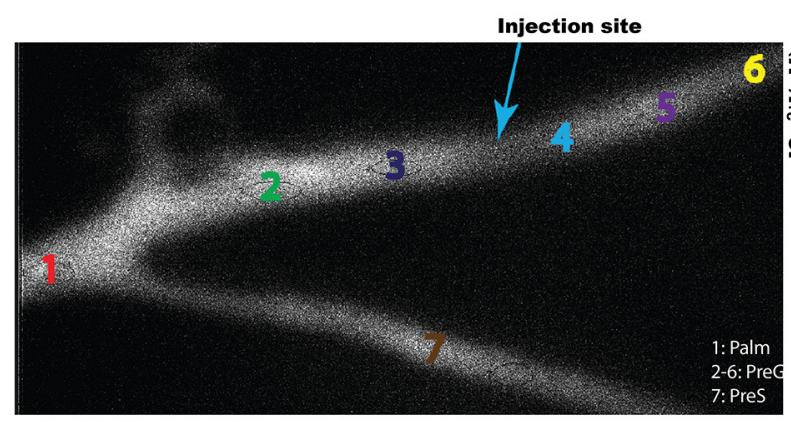

C

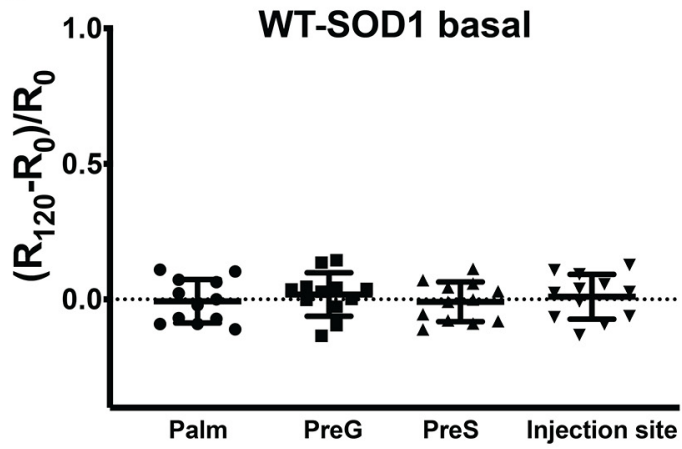

E

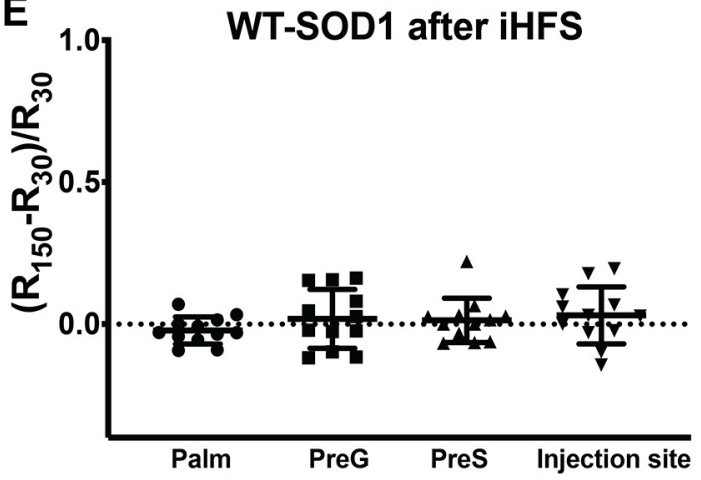

B

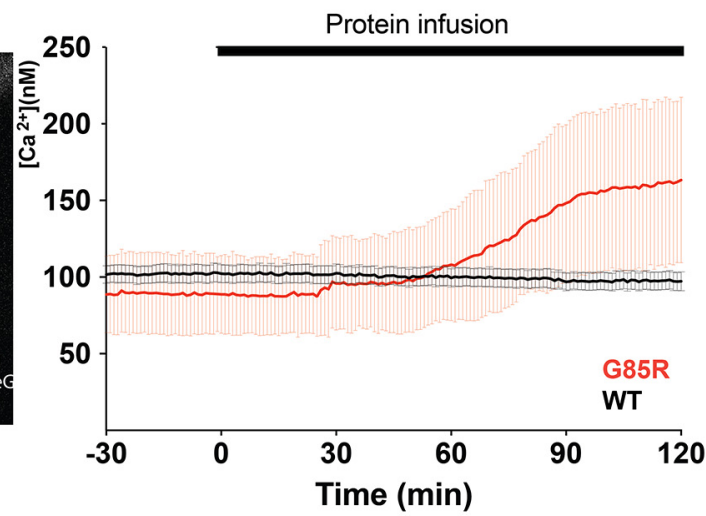

D

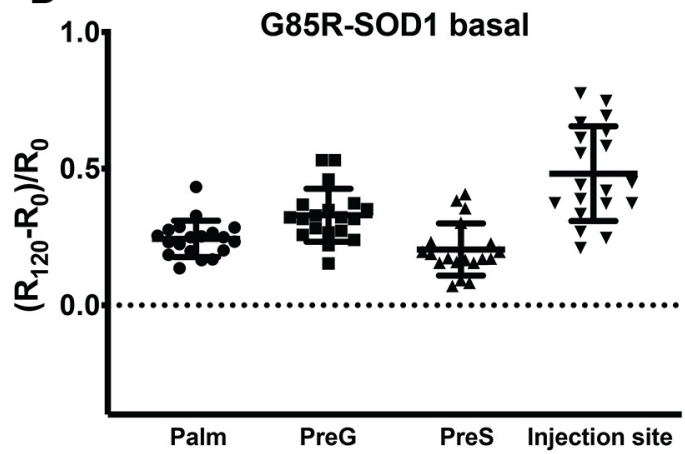

F

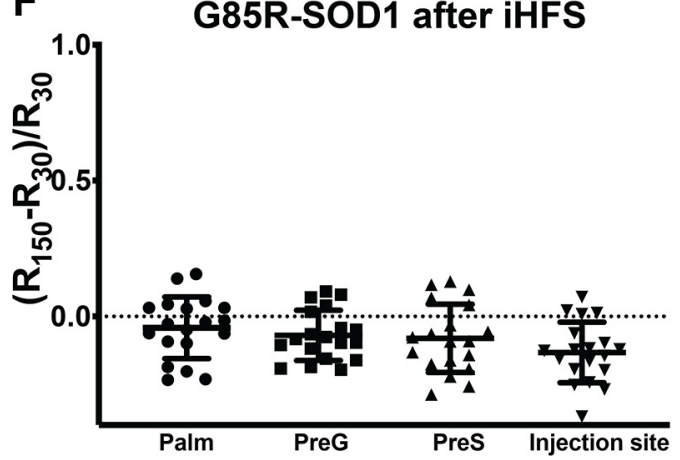

Figure 5. Presynaptic infusion of G85R-SOD1-His increased $\mathrm{Ca}^{2+}$ levels in the presynaptic terminal. Ratiometric live Ca ${ }^{2+}$ imaging was performed every $30 \mathrm{~s}$ after electrophoretic (100 nA current) infusion of a Ca ${ }^{2+}$ indicator, $1 \mathrm{~mm}$ fura- 2 in $100 \mathrm{~mm} \mathrm{KCl}$, into the presynaptic axon through the first presynaptic stimulation electrode inserted into the palm (Fig. 1A). Fura-2 injection continued for 10 min, and the synapse was allowed to equilibrate for $30 \mathrm{~min}$ before SOD1 injection through the second electrode to ensure fura-2 was diffused evenly throughout the whole presynaptic terminal at roughly $100 \mu \mathrm{m}$. Ratiometric images were taken at Ex360 nm and Ex390 nm assisted by ultra-high-speed wavelength switching $\lambda$ DG-4/5 xenon arc lamp system. Ratios at various locations $(\boldsymbol{A})$ were calculated as (fluorescent intensity $\left.{ }^{360 \mathrm{~nm}}\right) /\left(\right.$ fluorescent intensity ${ }^{390 \mathrm{~nm}}$ ) to provide a measure of intracellular $\mathrm{Ca}^{2+}$ concentrations. The blue arrow indicates the injection site for SOD1 proteins. 1: palm, 2-6: PreG, 4: infusion site, 7: PreS, an adjacent presynaptic axon branch which is smaller in size. B, G85R-SOD1-His induced $\mathrm{Ca}^{2+}$ increases while WT-SOD1-His had no effect on $\mathrm{Ca}^{2+}$ concentration. Raw $\mathrm{Ca}^{2+}$ concentrations were derived from the equation $\left[\mathrm{Ca}^{2+}\right]=\mathrm{Kd}^{\prime}(\mathrm{R}-\mathrm{Rmin}) /(\mathrm{Rmax}-\mathrm{R})$ and averaged across $12 \mathrm{WT}$ - and 19 G85R-SOD1-injected presynaptic terminals (PreG) respectively. Kd', Rmin, and Rmax were calculated from standards as described in Materials and Methods. Although different synapses varied in their baseline $\mathrm{Ca}^{2+}$ concentrations, G85R consistently increased $\left[\mathrm{Ca}^{2+}\right]$ at around 60 min after protein injection whereas WT had no effect. $\boldsymbol{C}, \boldsymbol{D}$, Baseline normalized $C^{2+}{ }^{2}$ ratio defined as $\left(R_{120}-R_{0}\right) / R_{0}\left(R_{120}: 60\right.$ min after SOD1 injection, $R_{0}$ : before SOD1 injection) were plotted at four sites for WT $(n=12)$ and G85R $(n=19)$. G85R-SOD1 caused increases in [Ca $\left.{ }^{2+}\right]$ at all sites including the palm where $\mathrm{Ca}^{2+}$ channels are sparse or absent. The increase in $\left[\mathrm{Ca}^{2+}\right]$ correlated roughly with G85R-SOD1 concentration, with the highest levels at the injection site and lowest in PreS, a smaller presynaptic axonal branch infused with fura-2 at a comparable concentration but with a lower SOD1 concentration due to slower diffusion from the injection site in PreG. $\boldsymbol{E}$, $\boldsymbol{F}$, iHFS applied at 30 min after SOD1 injection seemed to restore $\mathrm{Ca}^{2+}$ homeostasis in G85R-SOD1-injected synapses $(n=19)$, and this equilibrium lasted at least $2 \mathrm{~h}$ after iHFS, similar to that in WT $(n=12)$, this suggested the possibility that redistribution of $\mathrm{Ca}^{2+}$ is induced by iHFS. As expected, Ca ${ }^{2+}$ levels increased during HFS in synapses infused with either WT or G85R-SOD1 proteins (Extended Data Fig. 5-1). 
ratiometric $\mathrm{Ca}^{2+}$ imaging using fura-2, a fluorescent $\mathrm{Ca}^{2+}$ indicator dye, which was delivered to the presynaptic axon electrophoretically and allowed to diffuse into the palm and presynaptic branches [Fig. $5 A$, regions of interest (ROls) were selected to show 1: palm, 2-6: PreG, presynaptic axon which forms the giant synapse, and 7 : PreS, another small presynaptic branch]. In parallel, the presynaptic axon was subjected to basal stimulation while both presynaptic and postsynaptic membrane potentials were recorded. The presence of fura-2 provides an indication of changes in $\mathrm{Ca}^{2+}$ levels and location during stimulation. To avoid interference with $\mathrm{Ca}^{2+}$ imaging, WT-SOD1 and G85R-SOD1 constructs used in these studies lacked the YFP tag and injections were monitored by co-perfusion of tetramethylrhodamine-dextran. While overall characteristics of synaptic function are similar with the introduction of fura-2, the impairment of synaptic transmission by G85R-SOD1 was delayed in the presence of fura-2 ( $>60 \mathrm{~min}$, previously $<30 \mathrm{~min}$ after G85R-SOD1 injection), probably due to the buffering of $\mathrm{Ca}^{2+}$ by indicator dye. This impairment was correlated with a concurrent rise in $\mathrm{Ca}^{2+}$ levels at $60 \mathrm{~min}$. Synapses injected with fura2 alone or tetramethylrhodamine-dextran alone kept constant EPSP normally for $>2 \mathrm{~h}$ as those injected with WT. Furthermore, synapses injected with WT-SOD1 behaved similarly to those with WT-SOD1-YFP, excluding the potential role of YFP tag on synaptic transmission.

G85R-SOD1 infusion $(n=19)$ caused increases of $\mathrm{Ca}^{2+}$ throughout the presynaptic terminal compared with WT controls $(n=12)$, as derived from the ratios of (fluorescent intensity ${ }^{360 \mathrm{~nm}}$ )/(fluorescent intensity ${ }^{390 \mathrm{~nm}}$ ) averaged across ROls 2-6 (Fig. 5B). While WT-SOD1 under basal stimulation showed no change in $\mathrm{Ca}^{2+}$ concentration after $1 \mathrm{~h}$ protein infusion (Fig. 5C), G85R-SOD1 increased global $\mathrm{Ca}^{2+}$ concentration in the palm, the presynaptic giant axon (PreG), as well the other small presynaptic branch (PreS), with the most significant elevation at the site of G85RSOD1 injection (Fig. 5D) perhaps due to the highest G85RSOD1 concentration at the injection site. Both the palm and PreS regions, further away from the injection site, would contain lower concentrations of mutant protein due to diffusion. Interestingly, the palm which lacks plasma membrane $\mathrm{Ca}^{2+}$ channels and does not normally show $\mathrm{Ca}^{2+}$ increases during HFS did not exhibit increased $\mathrm{Ca}^{2+}$ with WT-SOD1 (Extended Data Fig. 5-1A), consistent with earlier findings (Smith et al., 1993). However, in the presence of G85R-SOD1, the palm also displayed aberrant $\mathrm{Ca}^{2+}$ increases with HFS (Extended Data Fig. 5-1B), suggesting that sources of $\mathrm{Ca}^{2+}$ other than plasma membrane $\mathrm{Ca}^{2+}$ channels may contribute to this $\mathrm{Ca}^{2+}$ misregulation. Taken together, the evidence suggests that G85R-SOD1induced $\mathrm{Ca}^{2+}$ influx may mediate its inhibitory effects on synaptic transmission and the effect may depend on intracellular $\mathrm{Ca}^{2+}$ stores.

Given that iHFS rescued G85R-SOD1-associated synaptic defects, we wondered if iHFS could correct the aberrant localization of $\mathrm{Ca}^{2+}$ increases. Fura-2 ratiometric $\mathrm{Ca}^{2+}$ imaging demonstrated $\mathrm{Ca}^{2+}$ increases during HFS in both WT- and G85R-SOD1-injected synapses (Extended Data Fig. 5-1), confirming previous findings of HFS on
$\mathrm{Ca}^{2+}$ dynamics by others (Smith et al., 1993). In the presence of G85R-SOD1, $\mathrm{Ca}^{2+}$ concentrations in the palm appeared more variable across the synapses, with some palm regions showing $\mathrm{Ca}^{2+}$ increases as high as those in the synaptic terminal, suggesting high baseline $\mathrm{Ca}^{2+}$ concentration. However, there seemed to be no significant difference between WT-SOD1-injected $(n=12)$ and G85RSOD1-injected $(n=19)$ synapses at the injection site and the other locations within the presynaptic terminals during iHFS, indicating normal $\mathrm{Ca}^{2+}$ channel functions at the injection site, thereby excluding the possibility of physical membrane damage caused by the injection. Consistent with iHFS rescue in synaptic physiology and in contrast to G85R-SOD1-induced $\mathrm{Ca}^{2+}$ influx in the absence of iHFS, aberrant $\mathrm{Ca}^{2+}$ activation was not observed after iHFS in G85R-SOD1-injected synapses (Fig. 5E,F). These results suggest that iHFS may reset $\mathrm{Ca}^{2+}$ dynamics in the terminal and prevent the local $\mathrm{Ca}^{2+}$ overloads that contribute to synaptic dysfunction induced by G85R-SOD1.

\section{EGTA prevents toxic effects of G85R-SOD1-YFP on synaptic physiology and morphology}

The iHFS rescue experiments in combination with $\mathrm{Ca}^{2+}$ imaging and the delayed inhibition produced by fura-2 suggested that aberrant $\mathrm{Ca}^{2+}$ activation by G85R-SOD1 may be the cause of synaptic inhibition. To further test this hypothesis, we chelated free $\mathrm{Ca}^{2+}$ in the presynaptic terminal by co-injecting $50 \mathrm{~mm}$ EGTA (final concentration at 5-10 $\mathrm{mm}$ in the synapse). Previous studies had shown that EGTA levels $<80 \mathrm{~mm}$ do not affect synaptic transmission (Adler et al., 1991). EGTA was sufficient to block the inhibitory effects of G85R-SOD1-YFP on synaptic transmission and G85R-SOD1-YFP-injected synapses maintained steady EPSPs for over $2 \mathrm{~h}$ without iHFS $(n=6)$, which was comparable to WT-SOD1-YFP-injected synapses (Fig. 6A; Extended Data Fig. 6-1; five WT-SOD1-infused and eight G85R-SOD1-infused synapses were also shown in Fig. $1 C)$. Not surprisingly, ratiometric $\mathrm{Ca}^{2+}$ imaging using fura2 did not show any local $\mathrm{Ca}^{2+}$ increases in synapses co-injected with G85R-SOD1 and EGTA (Fig. 6B,C). Defects in synaptic vesicle pools, number and distribution induced by G85R-SOD1 were also corrected by EGTA, as evidenced by the usual RRP size, and normal mobilization rate (Fig. $6 D, E)$. Furthermore, EM showed comparable numbers of synaptic vesicles at the AZs (Fig. 6F). The numbers of docked and electron lucent synaptic vesicles were similar between the WT-SOD1 and G85R-SOD1+EGTA-infused synapses, while EGTA might slightly increase clathrin coating (Fig. 6G; 102 synapses for each group). These results suggest that $\mathrm{Ca}^{2+}$ dysregulation may underlie G85RSOD1-associated synaptic dysfunctions and that limiting increases in free cytoplasmic $\mathrm{Ca}^{2+}$ with EGTA may restore normal synaptic morphology and function.

\section{Discussion}

The discovery of giant fibers in the squid nervous system by J. Z. Young (Young and Keynes, 2005) >80 years ago led to several milestone discoveries in modern neuroscience, including the Nobel-prize-winning work on ionic mechanisms for initiating and propagating action 
A

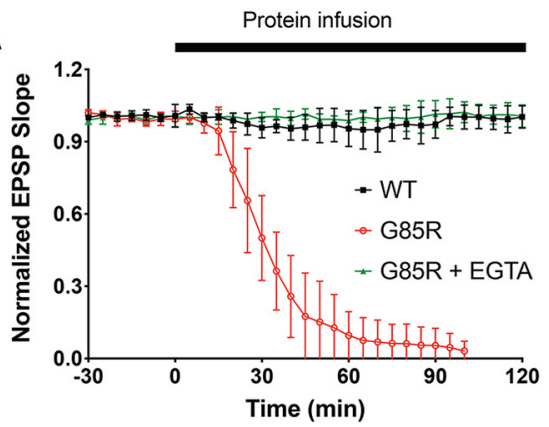

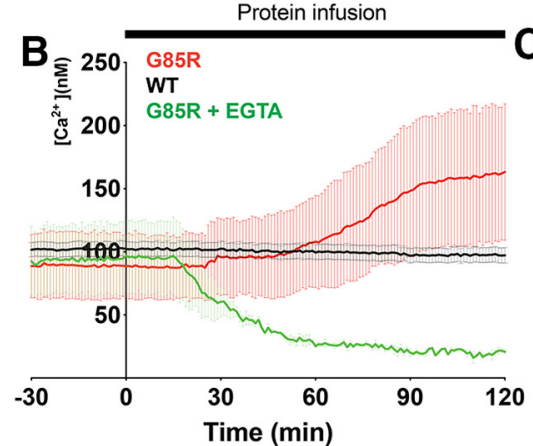

$\mathbf{F}$
E
D

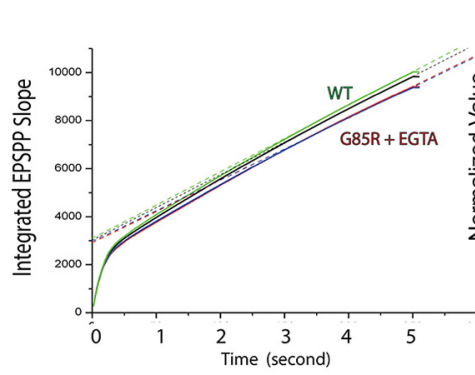

E
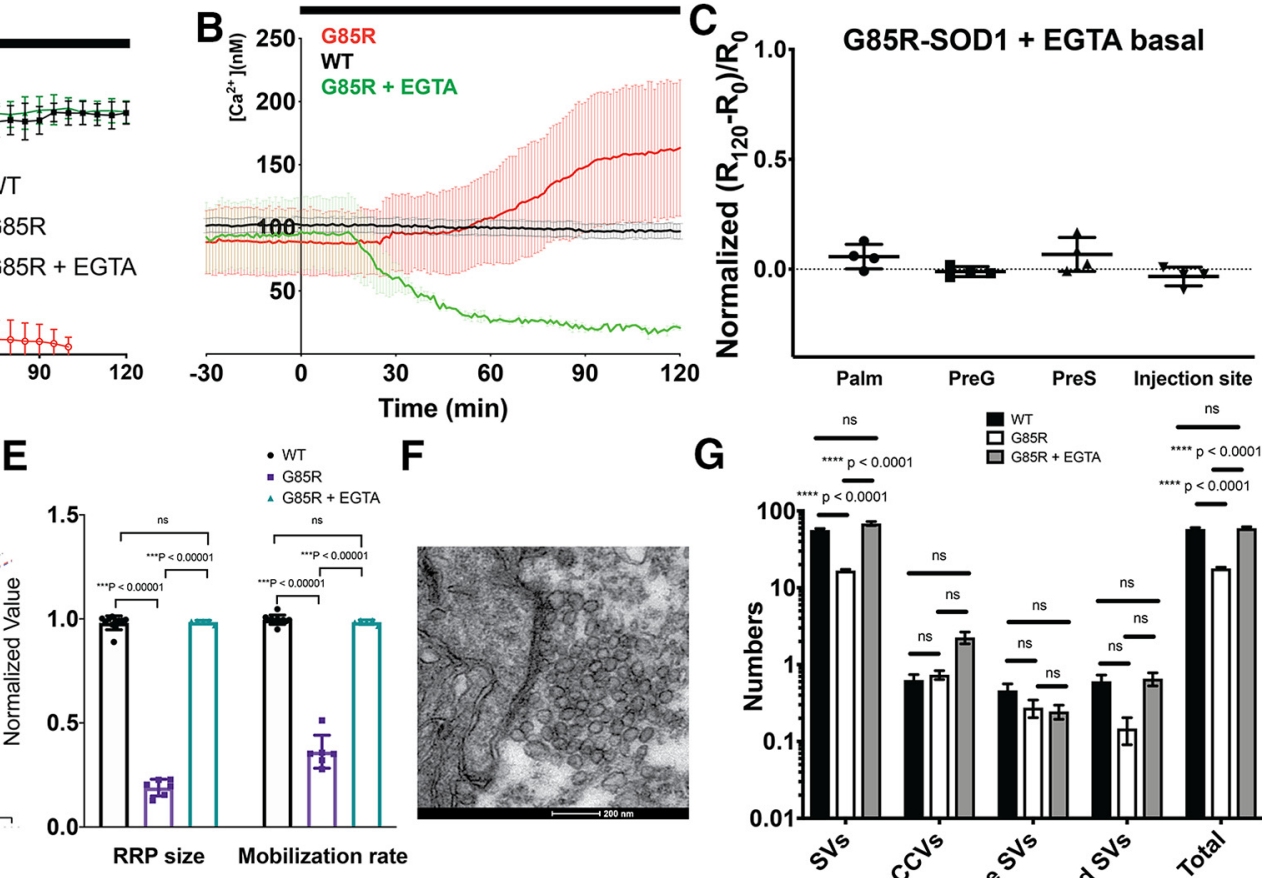

G

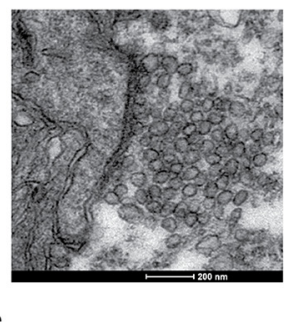

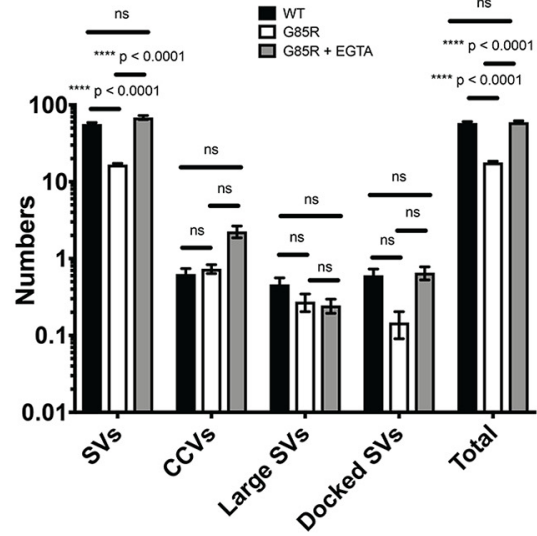

Figure 6. EGTA, a $\mathrm{Ca}^{2+}$ chelator, prevented G85R-SOD1-YFP-induced synaptic dysfunctions. A, EPSP slopes of six double injected synapses (50 mM EGTA and $50 \mu \mathrm{m}$ G85R-SOD1-YFP) were plotted from 30 min before the injection to $2 \mathrm{~h}$ after the injection. There was no reduction in EPSP slopes with EGTA and G85R-SOD1-YFP in contrast to that seen in synapses injected with G85R-SOD1YFP alone $(n=8)$ or WT-SOD1-YFP alone $(n=5)$, data for G85R-SOD1 and WT-SOD1 from Figure $1 C$ is included for comparison. All EPSP slopes were averaged and plotted with error bars indicating SE (for unnormalized data, see Extended Data Fig. 6-1). B, Ratiometric fura-2 $\mathrm{Ca}^{2+}$ imaging showed comparisons of $\mathrm{Ca}^{2+}$ concentrations in the presynaptic terminals (PreG) injected with WTSOD1, G85R-SOD1, and G85R-SOD1+ EGTA, EGTA prevented the $\mathrm{Ca}^{2+}$ increase caused by G85R-SOD1 ( $\left.n=4\right)$. Because of the chelating effect of EGTA, the basal level of $\mathrm{Ca}^{2+}$ decreased over time during co-infusion of G85R and EGTA. $\boldsymbol{C}$, Normalized ratios (subtracting levels with EGTA alone), positively correlated with $\mathrm{Ca}^{2+}$ concentrations, showed constant basal $\mathrm{Ca}^{2+}$ levels at various presynaptic regions injected with G85R-SOD1 in the presence of EGTA. $\boldsymbol{D}$, EPSP slopes from synapses injected with EGTA and G85R-SOD1 were integrated, normalized to the initial time point before the injection, and fit linearly to show RRP size as indicated by the intersection with the $y$-axis and the vesicle mobilization rate from RP to RRP as indicated by the slope of the linear fit. Compared with WT-SOD1-injected synapses (green) and synapses before injections (black), EGTA+G85R-SOD1-injected synapses showed little change in the RRP size and the mobilization $120 \mathrm{~min}$ after injection (red) and $180 \mathrm{~min}$ after injection (blue). E, Quantification of RRP size and mobilization for synapses injected with WT-SOD1 $(n=11)$, or G85R-SOD1 $(n=6)$, or G85R-SOD1 + EGTA $(n=5)$ showed that EGTA prevented the decreases in RRP size and mobilization rate caused by G85R-SOD1-YFP. Error bars indicate SE. $\boldsymbol{F}$, Representative EM image showed abundant synaptic vesicles at the AZ of a synapse injected with EGTA and G85R-SOD1-YFP. G, EGTA restored the synaptic vesicle numbers to normal at the AZs (102 AZs from three independent experiments for each group) ns: not significant.

potentials by Andrew Huxley and Alan Hodgkin (Schwiening, 2012). Although advanced imaging and genetic tools as well as new model systems have been developed in recent years, these classical mature synapse and axon models continue to be used to understand fundamental neurobiology and provide a unique model to study molecular mechanisms of human neurologic diseases. For example, the original discovery of the molecular motor kinesin in isolated axoplasm from the squid led to studies of axonal trafficking deficits resulting from altered motor function in several neurodegenerative diseases (Song et al., 2016; Brady and Morfini, 2017). In a similar way, studies of molecular mechanisms underlying basic synaptic physiology in the SGS have led to exploration of disease mechanisms involving synaptic dysfunction (Moreno et al., 2009). Here, we use the giant axoaxonic synapse in the squid stellate ganglion to study synaptic actions of mutant human SOD1 (G85R-SOD1) protein, which causes a familial form of ALS.

\section{Presynaptic injection of G85R SOD1 inhibits neurotransmission by disturbing synaptic vesicle physiology}

Synaptic loss at affected neuromuscular junctions leading to a dying-back neuropathy appears to be a primary defect associated with pathology in ALS patients and mouse models bearing G85R-SOD1 mutations. Consistent with these observations, we found that infusion of ALS-associated human G85R mutant SOD1 protein but not the WT-SOD1 into the presynaptic terminal of the SGS significantly inhibited EPSPs, leading to a failure in eliciting postsynaptic action potentials. This occurred within 30 min of continuous infusion into the presynaptic terminal without diminishing 
the presynaptic action potentials, while infusion of the WTSOD1 had no effect on synaptic transmission as evidenced by sustained firing of synapses with constant EPSPs (Fig. 1).

High-frequency stimulus protocols designed to deplete the RRP of presynaptic vesicles showed that vesicle dynamics were significantly inhibited by G85R-SOD1, reducing both the size of the RRP and the mobilization rate from the RP to the RRP (Fig. 2). This finding was further validated by EM data that demonstrated significant reductions of synaptic vesicles at AZs in the presence of G85R-SOD1 protein (Fig. 3). Ultrastructural studies provided additional evidence of altered vesicle trafficking as membranous structures resembling multivesicular bodies and autophagosomes were identified in presynaptic regions infused with G85R-SOD1, but not WT-SOD1. Inhibition of synaptic transmission was reversed by stopping presynaptic infusion of G85R-SOD1 and allowing sufficient time for the protein to diffuse out of the synapse via the presynaptic giant axon (Extended Data Fig. 1-2), which suggested that the synaptic machinery was not physically and permanently damaged.

\section{Nature of synaptic vesicle disturbance}

A clue to the mechanism for G85R-SOD1-induced synaptic inhibition came from an experiment where the synapse was exposed to iHFS at 30-min intervals before and during G85R-SOD1 infusion. This was expected to inhibit synaptic transmission based on earlier results showing that continuous HFS depleted presynaptic RRP. Unexpectedly, G85R-SOD1 synapses exposed to iHFS maintained constant EPSPs for over $7 \mathrm{~h}$, exhibiting normal synaptic vesicle dynamics and normal numbers of synaptic vesicles at AZs despite the presence of pathogenic SOD1 protein (Fig. 4). This finding led us to examine $\mathrm{Ca}^{2+}$ dynamics as HFS is known to redistribute $\mathrm{Ca}^{2+}$ both temporally and spatially (Smith et al., 1993).

Live cell ratiometric $\mathrm{Ca}^{2+}$ imaging studies showed that G85R-SOD1 consistently increased $\mathrm{Ca}^{2+}$ levels in presynaptic terminals with the largest increases at the injection site, the site with highest G85R-SOD1 concentration. Surprisingly, $\mathrm{Ca}^{2+}$ levels were also increased in the palm region, which lacks plasmalemmal $\mathrm{Ca}^{2+}$ channels and normally does not exhibit $\mathrm{Ca}^{2+}$ increases in response to HFS (Fig. 5). These data suggested that the source of $\mathrm{Ca}^{2+}$ may not be restricted to or dependent on local plasma membrane $\mathrm{Ca}^{2+}$ channels. This agrees with previous findings that $\mathrm{Ca}^{2+}$ influx solely through voltage-gated $\mathrm{Ca}^{2+}$ channels is not able to induce motor neuron death (Van den Bosch et al., 2002). Instead, release of $\mathrm{Ca}^{2+}$ from endoplasmic reticulum and/or mitochondrial stores is required, and these organelles may release $\mathrm{Ca}^{2+}$ in response to misfolded G85R-SOD1 protein.

\section{Co-injection of EGTA and application of iHFS prevent physiopathology}

If increased free $\mathrm{Ca}^{2+}$ levels are responsible for synaptic failure, co-injection of EGTA, a Ca ${ }^{2+}$ chelator, at levels that do not affect synaptic transmission at the SGS should prevent synaptic inhibition by G85R-SOD1 by buffering free $\mathrm{Ca}^{2+}$. Consistent with the conclusion that presynaptic increases in free $\mathrm{Ca}^{2+}$ levels were responsible for the observed synaptic dysfunction, EGTA prevented inhibition of synaptic transmission when co-injected into the presynaptic terminal with G85R-SOD1, as documented by (1) EPSPs remained constant for over $2 \mathrm{~h}$; (2) sufficient numbers of synaptic vesicle were present at the AZs with normal mobilization from RP to RRP; and (3) cytoplasmic free $\mathrm{Ca}^{2+}$ levels did not increase (Fig. 6). Similar effects were also achieved by iHFS applied every $30 \mathrm{~min}$ for over $7 \mathrm{~h}$ (Fig. 4). Interestingly, iHFS also abolished the $\mathrm{Ca}^{2+}$ increases induced by G85R-SOD1 (Fig. 5) perhaps by redistributing $\mathrm{Ca}^{2+}$ and restoring homeostasis. This process may alter regulation of other organelles such as ER, mitochondria, and autophagosomes in the synapse to restore overall synaptic health. However, determining the exact molecular mechanisms requires more in-depth studies.

Questions remain as to how increased cytoplasmic free $\mathrm{Ca}^{2+}$ alters synaptic vesicle dynamics in the presynaptic terminal. $\mathrm{Ca}^{2+}$ is a key player in many cellular and subcellular processes, is required for regular synaptic function, and is normally tightly regulated. In normal synapses, free $\mathrm{Ca}^{2+}$ increases are largely restricted to the AZ through local plasmalemmal channels during synaptic transmission (Sudhof, 2004). The levels of EGTA used in these studies do not affect $\mathrm{Ca}^{2+}$ influx at AZs but do buffer cytoplasmic free $\mathrm{Ca}^{2+}$. There are multiple $\mathrm{Ca}^{2+}$ sensors with a wide range of affinities in the presynaptic compartment, all of which may contribute to one or more of the complex steps involved in synaptic transmission and synaptic vesicle dynamics. While the role of some of these sensors is established, such as evidence that synaptotagmin is the $\mathrm{Ca}^{2+}$ sensor for synaptic vesicle fusion and neurotransmitter fast release, the physiological roles for many others remain unclear (Sudhof, 2004).

The existing literature has suggested a variety of potential mechanisms for $\mathrm{Ca}^{2+}$ related pathology in both human and mouse ALS-vulnerable neurons (Grosskreutz et al., 2010), such as the subgroups of motor neurons in spinal cord and brainstem expressing low levels of $\mathrm{Ca}^{2+}$ buffering proteins, even at the presymptomatic stages. Similarly, $\mathrm{Ca}^{2+}$ dysregulation has also been observed in several other neurodegenerative diseases and may lead to unfolded protein responses (UPRs) and ER stress-related signaling activation (Grosskreutz et al., 2010; Walker and Atkin, 2011; Hetz and Saxena, 2017; Remondelli and Renna, 2017), $\mathrm{Ca}^{2+}$ permeable AMPA receptor dysregulation (Tateno et al., 2004; Tortarolo et al., 2006; Van Den Bosch et al., 2006), ER/mitochondria membrane collapse (Tortarolo et al., 2006; Watanabe et al., 2016; BernardMarissal et al., 2018), and aberrant $\mathrm{Ca}^{2+}$ influx in affected neurons and glia. In addition, changes in protein expression in affected motor neurons (e.g., $\mathrm{Ca}^{2+}$ channels and regulators) may also contribute to dysregulation of $\mathrm{Ca}^{2+}$ levels in disease. While altered transcription may play a role in the slow progression of the disease, this is unlikely to contribute here given the time scale and reversibility of these effects as well as the lack of strong evidence of local transcription in the SGS.

In conclusion, our results in the SGS indicate that mutant SOD1 affects the regulation of free $\mathrm{Ca}^{2+}$ levels in the 
presynaptic terminal, leading to a disruption of normal vesicle trafficking. These effects were not restricted to regions near the AZs but were more extensive and affected regions of the presynaptic compartment that lack plasmalemmal $\mathrm{Ca}^{2+}$ channels. The effects of mutant SOD1 may occur without affecting the activity of local plasmalemmal $\mathrm{Ca}^{2+}$ channels required for neurotransmitter release at the $A Z$, given that (1) presynaptic regions lacking $\mathrm{Ca}^{2+}$ channels also showed increased levels of free $\mathrm{Ca}^{2+},(2)$ low levels of EGTA can reduce free $\mathrm{Ca}^{2+}$ levels in the presence of G85R-SOD1 without affecting $\mathrm{Ca}^{2+}$ influx through plasmalemmal $\mathrm{Ca}^{2+}$ channels responsible for normal synaptic transmission, and (3) the discovery that iHFS could rescue synaptic transmission. The effect of iHFS was unexpected and further argues for normal function of $\mathrm{AZ} \mathrm{Ca}^{2+}$ channels in the plasmalemma independent of intracellular stores of $\mathrm{Ca}^{2+}$. The effect of iHFS suggests that further study is needed of how patterns of activity can preserve synaptic function and this may be a basis for novel therapeutic interventions in ALS.

The effects observed here may relate to those observed earlier in the squid giant axon innervating muscles in the mantle. There we found that G85R-SOD1-YFP inhibited axonal trafficking of presynaptic vesicles by activating a MAPK stress pathway, which impaired kinesin-based transport responsible for anterograde cargo transport (Song et al., 2013). In addition, we observed that G85RSOD1 was associated with several synaptic proteins critical for synaptic transmission, such as synapsin and syntaxin-binding protein 5, at a much higher level than WTSOD1-YFP. HSP110, an HSC70 co-chaperone which prevented the neurotoxicity of G85R-SOD1 in the axon by potentially sequestering or refolding G85R-SOD1, also abolished the association of Synapse-associated protein and syntaxin-binding protein 5 with G85R-SOD1-YFP (Song et al., 2013). The roles that G85R-SOD1 interactions with synaptic proteins may play in affecting synaptic vesicle dynamics as well as regulation of these interactions via $\mathrm{Ca}^{2+}$ dependent or independent pathways remain to be determined. Various neuropathogenic changes have been proposed to be a consequence of $\mathrm{Ca}^{2+}$ dysregulation, such as UPR and autophagy, in ALS and several other neurodegenerative diseases. Our EM data (Fig. 6) showed that EGTA, when co-injected with G85R-SDO1, restored synaptic morphology and prevented the formation of pre-autophagosome and adjoining pre-lysosomes, which were observed when presynaptic G85R-SOD1 was injected alone.

\section{Implication of acute toxic effects of G85R-SOD1 on SGS physiology as relates to longer term ALS pathology in mammalian synapses}

Like many other adult-onset neurodegenerative diseases, one big puzzle in the field is "why does it take 4050 years to develop ALS?" Several disease-related processes occur during aging which can be overcome in young neurons due to robust compensatory mechanisms. In this study, we focus on acute effects of pathogenic SOD1, but a number of age-related factors may explain the delayed onset of clinical symptoms.
Unlike the acute injection of pathogenic SOD1 in this study where we observed the presence of pre-autophagosome and pre-lysosome formation within the time frame of both electrophysiological recordings and morphologic studies, ALS-associated mutant SOD1 in patients misfolds and accumulates gradually to pathologic levels over time, allowing adaptive changes in cellular and molecular mechanisms underlying synaptic transmission. This is due to, at least partially, mechanisms for actively removing pathogenic proteins in early stages of disease, which may be sufficient to slow the accumulation of toxic materials. However, these clearance mechanisms may eventually be unable to keep up with misfolded protein accumulations. By directly injecting defined levels of mutant SOD1 into an isolated SGS system, we ensured accumulation of misfolded protein locally in the synapses within a short period of time which limits compensatory effects in the isolated synapse with little protein degradation and synthesis in this time frame.

Similar to robust protein homeostatic pathways during development and maturation, $\mathrm{Ca}^{2+}$ buffering mechanisms and synaptic transmission may be redundant early on in the complex system such as the mammalian models where neuronal circuits (including the other cell types) are intact and dynamic. They can adapt to slowly increased stresses created by the presence of mutant SOD1, much as synaptic functions exhibit adaptive changes during normal aging. The use of isolated acute preparations of giant synapse also eliminates the possibility that transcriptional changes of synaptic proteins can compensate for the effects of toxic proteins on synaptic transmission machinery. For example, the question of whether ALS-affected neurons show hyper- or hypo- excitability has been debated for some time. As a matter of fact, several groups have reported hyperexcitability of motor neurons at early stages followed by hypoexcitability at later stages of ALS in both iPSC culture models and in mouse models. These changes may be due to various compensatory mechanisms at the molecular, cellular and systemic levels which also may contribute to the delayed onset of symptoms. Here, we can isolate inhibitory effects due to the simplicity of the squid synapse model system where many other factors, such as compensation from other neurons, changes in gene expression or axonal transport, or the impact of a complex environment can no longer play a role.

Furthermore, it is worth keeping in mind that synaptic loss, as a result of gradual decrease in synaptic strength as demonstrated here, occurs long before cell death and can be observed in presymptomatic stages of both ALS mice and patients. By measuring EPSP slopes and vesicle dynamics in the SGS, we have provided a sensitive readout of neurotransmission in real time and revealed changes in synaptic strength which are more subtle in pre-symptomatic patients and mouse models but are detectable long before clinically relevant behavioral abnormalities appear. Correlating $\mathrm{Ca}^{2+}$ dynamics and HFS effects with synaptic health in real time illuminates the kinetic changes in the presynaptic terminal with high temporal and spatial resolution, which suggests the importance of identifying potential 
therapeutic time windows. The acute effects demonstrated here may illustrate molecular mechanisms underlying disease pathology that are manifested over time in patients.

Relevant to mammalian physiology and pathology, the SGS also shares fundamental synaptic machineries with neuromuscular junctions in human, making it a unique ALS-disease model with a potential for answering important but otherwise difficult questions about pathogenic mechanisms and synaptic transmission in motor neuron diseases. The SGS provides a unique platform for (1) performing live measurements of both presynaptic and postsynaptic activities in response to disease-associated proteins with temporal and spatial resolutions surpassing those in most other model systems; (2) conducting morphologic studies (e.g. EM) in the same preparations characterized by electrophysiological studies and live-imaging of cellular and molecular pathways; (3) evaluating pharmacological inhibitors, biochemical tools, and electrophysiological protocols that may rescue disease phenotypes in a time dependent manner; and (4) identifying translatable mechanistic findings, due to the highly conserved synaptic machinery, that will provide better understanding and treatment of multiple neurodegenerative diseases where misfolded proteins cause disease pathology.

In conclusion, the development of novel models for study of neurodegenerative diseases such as those using the squid nervous system has the potential to provide unique insights into disease mechanisms. For example, the combination of our electrophysiological studies in the giant synapse with studies of axonal transport in isolated axoplasm from the postsynaptic giant axon of the squid, allowed us to identify signaling pathways altered by these pathogenic proteins (Song et al., 2013). These results were then developed further in mouse models of ALS and patient tissues or iPSCs. Our results here are also consistent with previous findings of $\mathrm{Ca}^{2+}$ dysregulation and synaptic dysfunction in both ALS mice and patient derived neurons (Grosskreutz et al., 2010; Tradewell et al., 2011; Leal and Gomes, 2015). For example, affected neurons exhibit aberrant $\mathrm{Ca}^{2+}$ dynamics, which leads to hyperexcitability or hypoexcitability at various stages (Wainger et al., 2014; Devlin et al., 2015; Martínez-Silva et al., 2018). Further, oculomotor neurons, which are relatively spared in ALS, have a higher buffering capacity for $\mathrm{Ca}^{2+}$ (Vanselow and Keller, 2000; Obál et al., 2006), which was thought to contribute to their overall resistance to pathology in patients and mouse models. Those data were unclear, however, as to whether these changes involved plasmalemmal $\mathrm{Ca}^{2+}$ channels or intracellular $\mathrm{Ca}^{2+}$ stores or both, an issue that could be addressed in the SGS.

Given recent work on the squid genome and improved mariculture protocols that have the potential to allow systematic long-term studies of squid nervous systems, there will be opportunities to develop novel transgenic ALS squid models. Therefore, studies using the SGS will continue to complement studies using human iPSCs and animal models of ALS as we seek to understand how multiple disease related pathways can independently affect the pleiotropic neuronal functions that interact to produce the phenotype of ALS, a multifactorial disease with a complex disease pathology.

\section{References}

Adler EM, Augustine GJ, Duffy SN, Charlton MP (1991) Alien intracellular calcium chelators attenuate neurotransmitter release at the squid giant synapse. J Neurosci 11:1496-1507.

Augustine GJ (1990) Regulation of transmitter release at the squid giant synapse by presynaptic delayed rectifier potassium current. J Physiol 431:343-364.

Augustine GJ, Charlton MP (1986) Calcium dependence of presynaptic calcium current and post-synaptic response at the squid giant synapse. J Physiol 381:619-640.

Augustine GJ, Charlton MP, Horn R (1988) Role of calcium-activated potassium channels in transmitter release at the squid giant synapse. J Physiol 398:149-164.

Augustine GJ, Morgan JR, Villalba-Galea CA, Jin S, Prasad K, Lafer EM (2006) Clathrin and synaptic vesicle endocytosis: studies at the squid giant synapse. Biochem Soc Trans 34:68-72.

Bernard-Marissal N, Chrast R, Schneider BL (2018) Endoplasmic reticulum and mitochondria in diseases of motor and sensory neurons: a broken relationship? Cell Death Dis 9:333.

Bloedel J, Gage PW, Llinás R, Quastel DM (1966) Transmitter release at the squid giant synapse in the presence of tetrodotoxin. Nature 212:49-50.

Brady ST, Morfini GA (2017) Regulation of motor proteins, axonal transport deficits and adult-onset neurodegenerative diseases. Neurobiol Dis 105:273-282.

Bruijn LI, Houseweart MK, Kato S, Anderson KL, Anderson SD, Ohama E, Reaume AG, Scott RW, Cleveland DW (1998) Aggregation and motor neuron toxicity of an ALS-linked SOD1 mutant independent from wild-type SOD1. Science 281:1851-1854.

Bunton-Stasyshyn RK, Saccon RA, Fratta P, Fisher EM (2015) SOD1 function and its implications for amyotrophic lateral sclerosis pathology: new and renascent themes. Neuroscientist 21:519-529.

Devlin AC, Burr K, Borooah S, Foster JD, Cleary EM, Geti I, Vallier L, Shaw CE, Chandran S, Miles GB (2015) Human iPSC-derived motoneurons harbouring TARDBP or C9ORF72 ALS mutations are dysfunctional despite maintaining viability. Nat Commun 6:5999.

Fischer LR, Culver DG, Tennant P, Davis AA, Wang M, CastellanoSanchez A, Khan J, Polak MA, Glass JD (2004) Amyotrophic lateral sclerosis is a distal axonopathy: evidence in mice and man. Exp Neurol 185:232-240.

Frey D, Schneider C, Xu L, Borg J, Spooren W, Caroni P (2000) Early and selective loss of neuromuscular synapse subtypes with low sprouting competence in motoneuron diseases. J Neurosci 20:2534-2542.

Fukuda M, Moreira JE, Lewis FM, Sugimori M, Niinobe M, Mikoshiba K, Llinas R (1995) Role of the C2B domain of synaptotagmin in vesicular release and recycling as determined by specific antibody injection into the squid giant synapse preterminal. Proc Natl Acad Sci USA 92:10708-10712.

Ghasemi M, Brown RH Jr (2018) Genetics of amyotrophic lateral sclerosis. Cold Spring Harb Perspect Med 8:a024125.

Grosskreutz J, Van Den Bosch L, Keller BU (2010) Calcium dysregulation in amyotrophic lateral sclerosis. Cell Calcium 47:165-174.

Hegedus J, Putman CT, Gordon T (2007) Time course of preferential motor unit loss in the SOD1 G93A mouse model of amyotrophic lateral sclerosis. Neurobiol Dis 28:154-164.

Hetz C, Saxena S (2017) ER stress and the unfolded protein response in neurodegeneration. Nat Rev Neurol 13:477-491.

Katz B, Miledi R (1967) A study of synaptic transmission in the absence of nerve impulses. J Physiol 192:407-436.

Katz B, Miledi R (1977) Suppression of transmitter release at the neuromuscular junction. Proc R Soc Lond B Biol Sci 196:465-469.

Leal SS, Gomes CM (2015) Calcium dysregulation links ALS defective proteins and motor neuron selective vulnerability. Front Cell Neurosci 9:225. 
Lin JW, Sugimori M, Llinas RR, McGuinness TL, Greengard P (1990) Effects of synapsin I and calcium/calmodulin-dependent protein kinase II on spontaneous neurotransmitter release in the squid giant synapse. Proc Natl Acad Sci USA 87:8257-8261.

Llinas R, Steinberg IZ, Walton K (1980) Transmission in the squid giant synapse: a model based on voltage clamp studies. J Physiol 76:413-418.

Llinas R, Steinberg IZ, Walton K (1981) Presynaptic calcium currents in squid giant synapse. Biophys J 33:289-321.

Llinás R, McGuinness TL, Leonard CS, Sugimori M, Greengard P (1985) Intraterminal injection of synapsin I or calcium/calmodulindependent protein kinase II alters neurotransmitter release at the squid giant synapse. Proc Natl Acad Sci USA 82:3035-3039.

Llinas RR, Sugimori M, Silver RB (1994) Localization of calcium concentration microdomains at the active zone in the squid giant synapse. Adv Second Messenger Phosphoprotein Res 29:133-137.

Martínez-Silva ML, Imhoff-Manuel RD, Sharma A, Heckman CJ, Shneider NA, Roselli F, Zytnicki D, Manuel M (2018) Hypoexcitability precedes denervation in the large fast-contracting motor units in two unrelated mouse models of ALS. Elife 7:e30955.

Moloney EB, de Winter F, Verhaagen J (2014) ALS as a distal axonopathy: molecular mechanisms affecting neuromuscular junction stability in the presymptomatic stages of the disease. Front Neurosci 8:252.

Moreno H, Yu E, Pigino G, Hernandez Al, Kim N, Moreira JE, Sugimori M, Llinas RR (2009) Synaptic transmission block by presynaptic injection of oligomeric amyloid beta. Proc Natl Acad Sci USA 106:5901-5906.

Morgan JR, Zhao X, Womack M, Prasad K, Augustine GJ, Lafer EM (1999) A role for the clathrin assembly domain of AP180 in synaptic vesicle endocytosis. J Neurosci 19:10201-10212.

Obál I, Engelhardt JI, Siklós L (2006) Axotomy induces contrasting changes in calcium and calcium-binding proteins in oculomotor and hypoglossal nuclei of Balb/c mice. J Comp Neurol 499:17-32.

Pasinelli P, Belford ME, Lennon N, Bacskai BJ, Hyman BT, Trotti D, Brown RH Jr (2004) Amyotrophic lateral sclerosis-associated SOD1 mutant proteins bind and aggregate with $\mathrm{Bcl}-2$ in spinal cord mitochondria. Neuron 43:19-30.

Remondelli P, Renna M (2017) The endoplasmic reticulum unfolded protein response in neurodegenerative disorders and its potential therapeutic significance. Front Mol Neurosci 10:187.

Sakowski SA, Lunn JS, Busta AS, Oh SS, Zamora-Berridi G, Palmer M, Rosenberg AA, Philip SG, Dowling JJ, Feldman EL (2012) Neuromuscular effects of G93A-SOD1 expression in zebrafish. Mol Neurodegener 7:44.

Schwiening CJ (2012) A brief historical perspective: Hodgkin and Huxley. J Physiol 590:2571-2575.

Smith SJ, Buchanan J, Osses LR, Charlton MP, Augustine GJ (1993) The spatial distribution of calcium signals in squid presynaptic terminals. J Physiol 472:573-593.

Song Y, Kang M, Morfini G, Brady ST (2016) Fast axonal transport in isolated axoplasm from the squid giant axon. Methods Cell Biol 131:331-348.

Song Y, Nagy M, Ni W, Tyagi NK, Fenton WA, Lopez-Giraldez F, Overton JD, Horwich AL, Brady ST (2013) Molecular chaperone Hsp110 rescues a vesicle transport defect produced by an ALSassociated mutant SOD1 protein in squid axoplasm. Proc Natl Acad Sci USA 110:5428-5433.

Sudhof TC (2004) The synaptic vesicle cycle. Annu Rev Neurosci 27:509-547.

Sugimori M, Tong CK, Fukuda M, Moreira JE, Kojima T, Mikoshiba K, Llinás R (1998) Presynaptic injection of syntaxin-specific antibodies blocks transmission in the squid giant synapse. Neuroscience 86:39-51.

Tateno M, Sadakata H, Tanaka M, Itohara S, Shin RM, Miura M, Masuda M, Aosaki T, Urushitani M, Misawa H, Takahashi R (2004) Calcium-permeable AMPA receptors promote misfolding of mutant SOD1 protein and development of amyotrophic lateral sclerosis in a transgenic mouse model. Hum Mol Genet 13:2183-2196.

Taylor JP, Brown RH Jr, Cleveland DW (2016) Decoding ALS: from genes to mechanism. Nature 539:197-206.

Tortarolo M, Grignaschi G, Calvaresi N, Zennaro E, Spaltro G, Colovic M, Fracasso C, Guiso G, Elger B, Schneider H, Seilheimer B, Caccia S, Bendotti C (2006) Glutamate AMPA receptors change in motor neurons of SOD1G93A transgenic mice and their inhibition by a noncompetitive antagonist ameliorates the progression of amytrophic lateral sclerosis-like disease. J Neurosci Res 83:134-146.

Tradewell ML, Cooper LA, Minotti S, Durham HD (2011) Calcium dysregulation, mitochondrial pathology and protein aggregation in a culture model of amyotrophic lateral sclerosis: mechanistic relationship and differential sensitivity to intervention. Neurobiol Dis 42:265-275

Urushitani M, Sik A, Sakurai T, Nukina N, Takahashi R, Julien JP (2006) Chromogranin-mediated secretion of mutant superoxide dismutase proteins linked to amyotrophic lateral sclerosis. Nat Neurosci 9:108-118.

Van den Bosch L, Van Damme P, Vleminckx V, Van Houtte E, Lemmens G, Missiaen L, Callewaert G, Robberecht W (2002) An alpha-mercaptoacrylic acid derivative (PD150606) inhibits selective motor neuron death via inhibition of kainate-induced $\mathrm{Ca} 2+$ influx and not via calpain inhibition. Neuropharmacology 42:706-713.

Van Den Bosch L, Van Damme P, Bogaert E, Robberecht W (2006) The role of excitotoxicity in the pathogenesis of amyotrophic lateral sclerosis. Biochim Biophys Acta 1762:1068-1082.

Vanselow BK, Keller BU (2000) Calcium dynamics and buffering in oculomotor neurones from mouse that are particularly resistant during amyotrophic lateral sclerosis (ALS)-related motoneurone disease. J Physiol 525:433-445.

Wainger BJ, Kiskinis E, Mellin C, Wiskow O, Han SS, Sandoe J, Perez NP, Williams LA, Lee S, Boulting G, Berry JD, Brown RH Jr, Cudkowicz ME, Bean BP, Eggan K, Woolf CJ (2014) Intrinsic membrane hyperexcitability of amyotrophic lateral sclerosis patient-derived motor neurons. Cell Rep 7:1-11.

Walker AK, Atkin JD (2011) Stress signaling from the endoplasmic reticulum: a central player in the pathogenesis of amyotrophic lateral sclerosis. IUBMB Life 63:754-763.

Wang J, Farr GW, Hall DH, Li F, Furtak K, Dreier L, Horwich AL (2009a) An ALS-linked mutant SOD1 produces a locomotor defect associated with aggregation and synaptic dysfunction when expressed in neurons of Caenorhabditis elegans. PLoS Genet 5: e1000350.

Wang J, Farr GW, Zeiss CJ, Rodriguez-Gil DJ, Wilson JH, Furtak K, Rutkowski DT, Kaufman RJ, Ruse CI, Yates JR 3rd, Perrin S, Feany MB, Horwich AL (2009b) Progressive aggregation despite chaperone associations of a mutant SOD1-YFP in transgenic mice that develop ALS. Proc Natl Acad Sci USA 106:1392-1397.

Watanabe S, Ilieva H, Tamada H, Nomura H, Komine O, Endo F, Jin S, Mancias P, Kiyama H, Yamanaka K (2016) Mitochondria-associated membrane collapse is a common pathomechanism in SIGMAR1- and SOD1-linked ALS. EMBO Mol Med 8:1421-1437.

Young JZ, Keynes R (2005) The Functioning of the Giant Nerve Fibres of the Squid. 1938 - J.Z. and the discovery of squid giant nerve fibres. J Exp Biol 208:179-180. 